\title{
3D-printing magnesium-polycaprolactone loaded with melatonin inhibits the development of osteosarcoma by regulating cell-in-cell structures
}

\author{
Weilin Zhang ${ }^{1 \dagger}$, Wei Zhao ${ }^{1 \dagger}$, Qin Li ${ }^{2}$, Duoyi Zhao ${ }^{1}$, Junxing Qu' ${ }^{1}$ Ziyang Yuan ${ }^{1}$, Zhihong Cheng ${ }^{1}$, Xiaojuan Zhu ${ }^{3}$,
} Xiuli Zhuang ${ }^{4}$ and Zhiyu Zhang ${ }^{1 *}$

\begin{abstract}
Melatonin has been proposed as a potent anticarcinogen presents a short half-life for osteosarcoma (OS). Cell-in-cell (CIC) structures play a role in the development of malignant tumors by changing the tumor cell energy metabolism. This study developed a melatonin-loaded 3D printed magnesium-polycaprolactone (Mg-PCL) scaffold and investigated its effect and molecular mechanism on CIC in OS. Mg-PCL scaffold was prepared by 3D-printing and its characteristic was determined. The effect and molecular mechanism of Mg-PCL scaffold as well as melatonin-loaded $\mathrm{Mg}-\mathrm{PCL}$ on OS growth and progression were investigated in vivo and in vitro. We found that melatonin receptor 1 (MT1) and CIC expressions were increased in OS tissues and cells. Melatonin treatment inhibit the key CIC pathway, Rho/ROCK, through the CAMP/PKA signaling pathway, interfering with the mitochondrial physiology of OS cells, and thus playing an anti-invasion and anti-metastasis role in OS. The Mg-PCL-MT could significantly inhibit distant organ metastasis of OS in the in vivo model. Our results showed that melatonin-loaded Mg-PCL scaffolds inhibited the proliferation, invasion and metastasis of OS cells through the CIC pathway. The Mg-PCL-MT could be a potential therapeutics for OS.
\end{abstract}

Keywords: Osteosarcoma, Melatonin, Cell-in-cell, Mg-PCL, Rho/ROCK, cAMP/PKA signaling pathway

\section{Introduction}

Osteosarcoma (OS), the most common type of malignant bone tumor [1], originates from the abnormal transformation of mesenchymal cells, and exhibits osteoblastic differentiation with the production of malignant bonelike substances [2]. OS is the most common bone cancer in adolescents and is one of the leading causes of death in children [3]. The incidence of OS is sex-related, with

\footnotetext{
*Correspondence: zyzhang@cmu.edu.cn

'Weilin Zhang and Wei Zhao-Co-first authors

1 Department of Orthopedics, The Fourth Affiliated Hospital of China

Medical University, Shenyang 110032, Liaoning, China

Full list of author information is available at the end of the article
}

a higher rate in male population [4], and mainly occurs in the metaphysis of long bones. Although complete radical excision of the extensive diseased extremity and en bloc resection of the tumor has been widely applied to treat OS, the mortality rate still remains high due to the high potential of metastasis [5]. Besides, implants need to be implanted when tumor resection causes bone defect and therefore, bone regeneration materials are attracted much attention.

Magnesium is the second most abundant caption in body and plays an essential role in metabolic function [6, 7]. More than half of the total Mg is distributed in bones [8]. It is reported that $\mathrm{Mg}$ ion is essential for new bone formation and could induce increase in osteogenic original author(s) and the source, provide a link to the Creative Commons licence, and indicate if changes were made. The images or other third party material in this article are included in the article's Creative Commons licence, unless indicated otherwise in a credit line to the material. If material is not included in the article's Creative Commons licence and your intended use is not permitted by statutory regulation or exceeds the permitted use, you will need to obtain permission directly from the copyright holder. To view a copy of this licence, visit http://creativecommons.org/licenses/by/4.0/. The Creative Commons Public Domain Dedication waiver (http://creativeco mmons.org/publicdomain/zero/1.0/) applies to the data made available in this article, unless otherwise stated in a credit line to the data. 
activity, as well as maintaining vascular function $[9,10]$. The Mg and its alloys are non-toxic, of excellent biodegradability, suitable mechanical compatibility, desirable osteoconductivity, biocompatibility and osseointegrative properties [11-13]. Therefore, they were considered as an ideal candidate for using as temporary, degradable orthopedic implants. However, $\mathrm{Mg}$ alloys are susceptible to corrosion in a biological environment [14], which is the main obstacle to their clinical use. Polycaprolactone (PCL) is a kind of synthetic polymer with a low degradation rate and desirable mechanical properties $[15,16]$. PCL has been utilized as a surface coating on Mg alloys in order to improve the corrosion resistance. Studies have shown that PCL has a slower degradation rate and exhibits biocompatibility on $\mathrm{Mg}[17,18]$. Mg-PCL composite offers an ideal candidate for bone tissue engineering with lower corrosion resistance and good biocompatibility.

Several cancer drug-loaded bone regeneration materials have been developed. Melatonin, a hormone secreted by the pineal gland [19], exerts physiological effects on the circadian rhythm, mood, sleep and aging [20]. Recent evidence has suggested that melatonin also has an impact on the treatment of many kinds of cancer, including OS, colon cancer, breast cancer, prostate cancer, and gastric cancer [21, 22]. It has been reported that melatonin could regulate bone formation, growth and differentiation as well as exert anti-osteoporosis effects [23, 24]. Because melatonin presents variable, low bioavailability and a short half-life, its clinical application is significantly hindered. Besides, systemic administration requires a large amount of melatonin, which might lead to drug side effects. Therefore, drug delivery system, such as polymeric nanoparticulated systems might provide promising alternatives [25].

Previous studies have reported that melatonin can affect intracellular cAMP level by acting on melatonin receptor 1 (MT1) [26], and it has been reported that cAMP regulates the formation of Cell-in-cell (CIC) formation through the Rho/ROCK pathway [27]. CIC structures refer to the phenomenon whereby one or more living cells are internalized into adjacent living cells [28, 29]. The CIC process is caused by the loss of matrix adhesion, leading to invasion of one or more living cells into another. The discovery of CIC can be dated back to more than 100 years ago; however, its research only attracted great interest in recent years because of its potential pathophysiological significance [30]. CIC formation between tumor cells has been reported to alter energy metabolism, which closely related to the occurrence and development of tumors [31, 32]. Considerable evidence has suggested that the CIC phenomenon could promote tumor metastasis, except for pancreatic cancer and influence cancer prognosis [33, 34]. However, the effect of melatonin on CIC formation in OS has not been fully elucidated.

Three-dimensional (3D) printing technology is a precise, fast and controllable fabrication technology which fits the aim of personalized treatment nowadays [35]. 3D printed materials have been utilized into many fields [36]. In this study, we loaded melatonin in a 3D printing $\mathrm{Mg}-\mathrm{PCL}$ scaffold and investigated its effects and molecular mechanism on inhibiting OS growth and metastasis. We hypothesized that the melatonin-loaded $\mathrm{Mg}-\mathrm{PCL}$ implants affects CIC formation through the cAMP/Rho/ ROCK pathway and thereby inhibiting OS growth. Our results might provide a new therapeutic strategy for treating OS.

\section{Methods}

\section{Patients}

From February 2015 to December 2018, 30 fresh OS patient tissue samples were collected from the Department of Orthopedics, the Fourth Affiliated Hospital of China Medical University. After collection, the samples were immediately stored in liquid nitrogen at $-80^{\circ} \mathrm{C}$ for future use. Detailed information of each sample is shown in Table 1. All procedures performed in studies involving human participants were in accordance with the ethical standards of the institutional and national research committee and with the 1964 Helsinki declaration and its later amendments or comparable ethical standards. Written informed consent has been received from all included patients and the research protocol has been approved by the Fourth Affiliated Hospital of China Medical University Ethics Committee (No. EC-2018-KS-058).

Table 1 Clinicopathological features of OA patients $(n=30)$

\begin{tabular}{lll}
\hline Characteristics & Number of OA patients & Percent (\%) \\
\hline Gender & 15 & 50 \\
Male & 15 & 50 \\
Female & & \\
Age, years & 17 & 56.7 \\
$\leq 25$ & 13 & 43.3 \\
$>25$ & & \\
Tumor site & 16 & 53.3 \\
Tibia & 6 & 20 \\
Femur & 8 & 26.7 \\
Other & & \\
TNM staging & 10 & 33.3 \\
I-II & 20 & 66.7 \\
III-IV & & \\
Distant metastasis & 12 & 40 \\
Yes & 18 & 60 \\
No & &
\end{tabular}




\section{Reagents}

Pentanoic acid (HY-N6056), ROCK inhibitor-2 (HY119937), S26131 (HY-122136) and 7-Desmethyl-agomelatine (HY-133113) were obtained from Med Chem Express (MCE, New Jersey, USA). SunP Gel G1 was purchased from SunP Biotech (Beijing, China). Bovine serum albumin (BSA) was purchased from Sigma-Aldrich. AntiE-cadherin (ab1416), anti-MTNR1A (ab203038), antiMTNR1B (ab203346), anti-tubulin (ab210797), Alexa 488 (ab150077) and Alexa 647 (ab150115) were purchased from Abcam (Cambridge, UK). Anti-COX4I1 (11242-1AP) and anti-ATPB (17247-1-AP) were purchased from Proteintech (Hubei, China). Anti-TOM20 (\#42406), antiPGC1 $\alpha$ (\#2178), anti-NRF1 (\#46743), and anti-TFAM (\#8076) were obtained from Cell Signaling Technology (Massachusetts, USA). Anti-glyceraldehyde-3-phosphate dehydrogenase (GAPDH, TA309157) and all secondary antibodies were purchased from Zsbio (Beijing, China).

\section{D-printing of $\mathrm{Mg}-\mathrm{PCL}$ scaffolds}

Different ratios (W/W) of Mg powder (1\%, 2.5\%, 5\% and $10 \%$ ) and PCL (MW: 80,000 Da) were weighed and mixed at high temperature with an internal mixer for $10 \mathrm{~min}$. The temperature was set at $120^{\circ} \mathrm{C}$ and the rotating speed was set at $20 \mathrm{rpm}$. After mixing, the material was cut into small particles. Scaffolds were printed by the 3D printer (BioMaker, Ubbiotech, China) using SolidWorks 2017 software and Simplify 3D software. Printing parameters were set as follows: printing temperature was $120^{\circ} \mathrm{C}$, filling rate was 50\%, and printing speed was $6 \mathrm{~mm} / \mathrm{s}$.

\section{Melatonin loading on the scaffold}

Melatonin and SunP Gel G1 were added into $10 \mathrm{ml}$ Phosphate Buffer Saline (PBS) with a mass ratio of 1:4, 1:3, $1: 2,1: 1$ and 2:1. The mixture was stirred at $650 \mathrm{r} / \mathrm{min}$ for $2 \mathrm{~h}$ in a water bath at $40{ }^{\circ} \mathrm{C}$ to completely dissolve. After being cooled to room temperature $\left(23-25^{\circ} \mathrm{C}\right)$, the MT-loaded gel was obtained. MT-loaded SunP Gel G1 gel $(300 \mu \mathrm{l})$ was injected into the gap of the 3D Mg-PCL scaffold. The scaffold was placed at room temperature $\left(23-25{ }^{\circ} \mathrm{C}\right)$ to achieve liquid-solid conversion to obtain MT-loaded Mg-PCL scaffold.

\section{Determination of characterisics of $\mathbf{M g}-\mathrm{PCL}$ scaffold}

Chemical composition of the scaffold was determined by Fourier transform infrared spectroscopy (FTIR, Perkin Elmer, FTIR-2000). For thermogravimetric analysis (TGA), samples were heated at $10{ }^{\circ} \mathrm{C} / \mathrm{min}$ in open aluminium pans with a Discovery TGA (TA instruments, Waters, LLC, USA). Nitrogen was used as a purge gas with a flow rate of $25 \mathrm{ml} / \mathrm{min}$. Data collection and analysis were performed using TA Instruments Trios software and \% mass loss and/or onset temperature were calculated. The wide-angle X-ray diffraction (XRD) pattern of chitin powders and the dried sheets were recorded on an XRD instrument (D8ADVANCE, BRUKER, Germany) with $\mathrm{Cu}-\mathrm{K}$ radiation $(\lambda=0.154 \mathrm{~nm})$. The XRD data were collected from $2 \theta=5$ to $35^{\circ}$ at a scanning rate of $2^{\circ}$ $\mathrm{min}^{-1}$. The mechanical properties of the scaffold were measured using a universal mechanical testing machine (Instron 1121, UK). Scanning electron microscopy (SEM) Surface and cross-section images of the filaments were taken with an SEM (JSM-840 A Scanning Microscope, JEOL GmbH, Eching, Germany).

\section{In vitro immersion test}

The samples were weighted and recorded. Then, the samples were placed into clean $15 \mathrm{ml}$ tubes. Hank's solution was then added to the tipped plastic bottle at a ratio of the surface area of the sample of $1 \mathrm{~cm}^{2}: 25 \mathrm{ml}$. The soaking bottle was placed in a WE-1 type water bath thermostat at $37^{\circ} \mathrm{C}$. $\mathrm{pH}$ was recorded every day. After 30 days, the samples were dried with a hair dryer. The surfaces were photographed under SEM. Afterwards, the samples were ultrasonically cleaned by chromic acid solution $\left(200 \mathrm{~g} / \mathrm{l} \mathrm{CrO}_{3}\right)$, distilled water and ethyl alcohol for $10 \mathrm{~min}$. The samples were weighted again after being dried by a hair dryer. The corrosion rate was calculated using the follow formula:

$$
\text { Corrosion rate }=(\mathrm{K} \times \mathrm{W}) /(\mathrm{A} \times \mathrm{T} \times \mathrm{D})
$$

where $\mathrm{K}$ equals $8.76 \times 10^{4}$; W is the weight difference before and after soaking (g); A is the surface area of the sample exposed to Hank's solution $\left(\mathrm{cm}^{2}\right) ; \mathrm{T}$ is the soak period (h) and D is the density of samples.

\section{Determination of loading efficiency (LE) and loading capacity (LC)}

Melatonin solution was prepared using absolute ethanol and chloroform ( $\mathrm{V}: \mathrm{V}=9: 1)$ as solvent. The wavelength of the maximum absorption peak was measured at the wavelength range of 200-350 nm using an ultravioletvisible spectrophotometer (Mettler Toledo, Switzerland). Standard curve was draw by linear regression fitting of the absorbance with the mass concentration of the melatonin solution at the wavelength of the maximum absorption peak $(278 \mathrm{~nm})$. Freeze-dried melatonin-loaded SunP Gel G1 gel (50 mg) was dissolved in PBS (10 ml). After being centrifugation at 12,000 rpm for $5 \mathrm{~min}, 0.5$ $\mathrm{ml}$ supernatant was diluted in PBS in a $25 \mathrm{ml}$ volumetric flack. The absorbance value of the solution was measured at $278 \mathrm{~nm}$. The mass of melatonin was calculated from the standard curve and the LE and LC was calculated by following formula: 


$$
\begin{aligned}
& \mathrm{LE}=\mathrm{m}_{\mathrm{a}} / \mathrm{m}_{\mathrm{b}} \times 100 \% \\
& \mathrm{LC}=\mathrm{m}_{\mathrm{a}} / \mathrm{m}_{\mathrm{c}} \times 100 \%
\end{aligned}
$$

where $\mathrm{m}_{\mathrm{a}}$ means the mass of melatonin in melatoninloaded SunP Gel G1 gel, $\mathrm{m}_{\mathrm{b}}$ represents total mass of melatonin and $m_{c}$ is the mass of SunP Gel G1 in melatonin-loaded SunP Gel G1 gel.

\section{In vitro release of melatonin from melatonin-loaded scaffolds}

Standard curve of melatonin was first draw using PBS $(\mathrm{pH}=7.4)$ as solvent under the wavelength of the maximum absorption peak (278 nm). Melatonin-loaded SunP Gel G1 gel (100 mg) was dissolved in PBS and placed in a dialysis bag. The dialysis bag was then placed in a beaker containing the same pH PBS buffer and was vibrated horizontally in a constant temperature shaker $\left(37{ }^{\circ} \mathrm{C} \pm 0.5{ }^{\circ} \mathrm{C}\right)$ with a vibration frequency of 50 times/ $\mathrm{min}$. The release liquid $(5 \mathrm{ml})$ was replaced with same amounts of fresh PBS at selected time intervals. The absorbance value of the solution was measured at $278 \mathrm{~nm}$ and the drug release rate was calculated as follow:

$$
\mathrm{R}(\%)=\frac{\rho_{n} V+V_{i} \sum_{t}^{n-i} \rho_{i}}{M_{D}} \times 100
$$

where $\mathrm{R}$ means the cumulative drug release rate (\%); $\mathrm{n}$ means the number of sampling. $\rho_{n}$ represents the mass concentration $(\mathrm{g} / \mathrm{l})$ of the drug in the nth release. $V$ represents the total volume of the release liquid. $\rho_{i}$ represents the mass concentration $(\mathrm{g} / \mathrm{l})$ of the drug in the ith release, $\mathrm{V}_{\mathrm{i}}$ represents the volume of the release liquid at the ith sampling and $M_{D}$ means the mass of drug loaded (g).

\section{Pharmacokinetics of melatonin}

The chromatographic conditions for the high-performance liquid chromatography (HPLC) analysis of melatonin were as follows: Hypersil ODS2 column (150 mm $\times 4.6 \mathrm{~mm}, 5 \mu \mathrm{m})$; mobile phase containing $20 \mathrm{mmol} \mathrm{l}^{-1}$ $\mathrm{NaAc-HAC}$ buffer solution $(\mathrm{pH}=3.4) /$ methanol $(65: 35$ $\mathrm{v} / \mathrm{v}$ ); flow rate of $1.0 \mathrm{ml} \mathrm{min}^{-1}$; excitation (Ex) and emission (Em) detection wavelengths of $285 \mathrm{~mm}$ and $345 \mathrm{~mm}$, respectively; and column temperature of $35^{\circ} \mathrm{C}$.

For sample treatment, $200 \mu \mathrm{l}$ of mice plasma was added into $1.2 \mathrm{ml}$ of ethyl acetate for extraction, and vortex mixed for $2 \mathrm{~min}$. Following centrifugation for $10 \mathrm{~min}$ $\left(150,000 \mathrm{R} \mathrm{min}^{-1}, 4{ }^{\circ} \mathrm{C}\right), 1 \mathrm{ml}$ of the ethyl acetate layer (upper layer) was transferred into another centrifuge tube, and dried with nitrogen. The residue was then redissolved in $100 \mu \mathrm{l}$ of mobile phase, vortex mixed for $30 \mathrm{~s}$, and centrifuged for $10 \mathrm{~min}\left(150,000 \mathrm{R} \mathrm{min}^{-1}\right)$, to allow 20 $\mu \mathrm{l}$ of the supernatant to be drawn for injection.

\section{Animal experiment of mice for OS growth}

Female Balb/c nude mice ( $\mathrm{n}=6,8$ weeks old) were purchased from the Experimental Animal Center of Jilin University. The mice were bred under temperature-controlled conditions at $22.6 \pm 2{ }^{\circ} \mathrm{C}$ with a 12 -h day/light cycle. All applicable institutional and national guidelines for the care and use of animals were followed and the animal experiments were approved by the Animal Care and Use Committee of China Medical University. The heterograft mouse model was established by subcutaneous injection of $2 \times 10^{8}$ human OS (U2OS) cells suspended in $0.2 \mathrm{ml}$ medium into the root of the right thigh.

When the tumors grew to about $80 \mathrm{~mm}^{3}$, the mice were randomly divided into 8 groups: (1) control group: mice received intraperitoneal treatment of PBS (vehicle); (2) MT group: mice received intraperitoneal injection of melatonin (40 mg kg $\mathrm{day}^{-1}$ ); (3) MT + ROCK activator group: mice received intraperitoneal injection of melatonin $\left(40 \mathrm{mg} \mathrm{kg}^{-1}\right.$ day $\left.^{-1}\right)$ and ROCK activator (10 mg kg $\mathrm{may}^{-1}$ ), respectively; (4) MT + ROCK inhibitor group: mice received intraperitoneal injection of melatonin $\left(40 \mathrm{mg} \mathrm{kg}^{-1} \mathrm{day}^{-1}\right)$ and ROCK inhibitor (10 $\mathrm{mg} \mathrm{kg}^{-1}$ day $^{-1}$ ), respectively; (5) $\mathrm{Mg}-\mathrm{PCL}$ group: Mg-PCL scaffold (10 mm *2.5mm * $2.5 \mathrm{~mm}$ ) was subcutaneous implanted into the root of the right thigh, next to the tumor; (6) Mg-PCL + MT group: melatonin was loaded into Mg-PCL scaffold with SunP Gel G1 and was subcutaneous implanted into the root of the right thigh, next to the tumor; (7) Mg-PCL + MT + ROCK activator group: MT-loaded Mg-PCL scaffold was subcutaneous implanted into the root of the right thigh, next to the tumor; mice received intraperitoneal injection of ROCK activator (10 mg kg day $\left.^{-1}\right)$; (8) Mg-PCL + MT + ROCK inhibitor group: MT-loaded Mg-PCL scaffold was subcutaneous implanted into the root of the right thigh, next to the tumor; mice received intraperitoneal injection of ROCK inhibitor $\left(10 \mathrm{mg} \mathrm{kg}^{-1} \mathrm{day}^{-1}\right)$. All treatments sustained for 27 days.

The mice were sacrificed. Tumors and main organs (heart, liver, spleen, lung and kidney) were collected, weighed, fixed overnight in $4 \%$ paraformaldehyde, and then embedded in paraffin. The paraffin-embedded samples were cut into a thickness of $5 \mu \mathrm{m}$ and used for the staining of hematoxylin and eosin (HE). Histological alterations were evaluated using a fluorescence microscope (Nikon Eclipse Ti, Optical Apparatus Co., Ardmore, USA) and a confocal microscope (Carl Zeiss, LSM 780).

\section{Animal experiment of rabbits for OS metastasis}

New Zealand white rabbits (2-month old) weighing 2.0$3.0 \mathrm{~kg}$ were purchased from the animal experiment center 
of China Medical University. VX2 sarcoma cell line was purchased from Shanghai Yubo Biotechnology Co., Ltd and the tumor was passaged by continuous passage of tumor-bearing rabbits. The tumor was excised from the lower limbs of the sacrificed tumor-bearing rabbits. After removed the blood vessels and fibrous tissue around the tumor tissue with ophthalmic scissors, the tumor was cut into small pieces and immersed in normal saline for use.

The rabbits were routinely anesthetized. The plane of the front edge of the proximal tibia of the rabbit was fully exposed. A $1.5 \mathrm{~cm}$ incision was made longitudinally on the tibia. The fascia and periosteum were peeled off to expose the tibial cortex. A $1 \mathrm{~mm}$ diameter hole was made in the cortex using a dental drill. Two to three pieces of tumor tissue with a volume of about $1 \mathrm{~mm}^{3}$ were inoculated into the medullary cavity. The orifice and the surrounding $1 \mathrm{~cm}$ area were sealed with bone wax. The fascia and skin were sutured sequentially. One month after the tumor was implanted and an incision was made layer by layer along the longitudinal axis of the tumor to expose the tibia, to verify the modeling of the OS model.

The OS model animals were divided into control group (without any implant), a titanium-PCL (Ti-PCL) group, a Mg-PCL implant group (Mg-PCL), and a Mg-PCL carrying melatonin group (Mg-PCL-MT). The bone tumor was completely removed. A hole was drilled at the front edge of the tibial plateau, and the implant was inserted into the bone marrow cavity. Two months after the operation, the experimental animals were sacrificed, the rabbit tibia was completely removed for MicroCT detection, and the rabbit organs (heart, liver, spleen, lung, and kidney) were taken for HE staining.

\section{HE staining}

Tissues were fixed in 4\% paraformaldehyde. After fixation, the samples were decalcified in 10\% EDTA for 21 days before paraffin embedding and then sliced microtomically into a systematic series of $16 \mu \mathrm{m}$ sections. Standard HE staining was performed and photographed. The average number of deposits per section was calculated using Image-Pro Plus 6.0 (Media Cybernetics, USA).

\section{Immumohistochemical staining}

Tumor sections were treated with $0.6 \% \mathrm{H}_{2} \mathrm{O}_{2}$ in PBS for $20 \mathrm{~min}$ and then washed with Tris-buffered saline (TBS) for three times. After that, sections were blocked in $0.5 \%$ Triton- $\mathrm{x}-100$ in TBS and Triton X-100/3\% horse serum (TBS-TS) for $60 \mathrm{~min}$ at room temperature $\left(23-25{ }^{\circ} \mathrm{C}\right)$, and incubated with primary antibody ki-67 at 1:400 in TBS-TS at $4{ }^{\circ} \mathrm{C}$ for $48 \mathrm{~h}$. Then after four times of washing with TBS, sections were incubated with secondary goatrabbit IgG antibodies (1:1000, Bioworld) at room temperature $\left(23-25^{\circ} \mathrm{C}\right)$ for $2 \mathrm{~h}$. After three times of washing with TBS, DAB solution was added to incubate for $5 \mathrm{~min}$. Images were obtained with a DP72 digital camera (Olympus, Tokyo, Japan) and DP2-BSW microscope digital camera software (Olympus).

\section{Tunnel assay}

Tunnel assay was performed according to the instructions of commercially available kit (Beyotime C1086). Tumor sections were fixed with h $4 \%$ paraformaldehyde at room temperature $\left(23-25{ }^{\circ} \mathrm{C}\right)$ for $15 \mathrm{~min}$. Fixed cells were permeabilized with $0.2 \%$ Triton $\mathrm{X}-100$ and then blocked with 5\% BSA in PBS. After these basic operations, sections were treated with tunnel staining according to the kit's specification. Images were obtained with a confocal laser scanning microscope (Olympus, Tokyo, Japan).

\section{MicroCT scan}

The tibias of rabbits or mice were aseptically removed and assessed by MicroCT scan as described previously [37]. The bone mineral density (BMD), trabecular number (Tb.N), trabecular bone volume per tissue volume (BV/TV) and trabecular thickness (Tb.Th) were determined.

\section{Cell culture}

The human fetal osteoblastic cell line, hFOB1.19, and OS cell line, U2OS, MG63, HOS and 143B, were purchased from the Cell Bank of the Chinese Academy of Sciences (Shanghai, China). The cells were suspended in a 1:1 mixture of Ham's F12 medium and Dulbecco's Modified Eagle's Medium (DMEM/F12) (HyClone, Utah, USA), $10 \%$ fetal bovine serum (HyClone) and $0.3 \mathrm{~g} / \mathrm{l} \mathrm{G418}$ (Sigma-Aldrich, Darmstadt, Germany), and maintained in a humidified $5 \% \mathrm{CO}_{2}$ atmosphere at $33.5{ }^{\circ} \mathrm{C}$. The medium was changed every 2 days, and the cells were passaged using Trypsin-EDTA (HyClone). The cells were plated at $10^{4}$ cells $/ \mathrm{cm}^{2}$ for subsequent experiments.

U2OS cells were divided into 8 groups: (1) Control group: without any treatment; (2) MT group: U2OS cells were added with $1 \mathrm{mM}$ melatonin; (3) MT + ROCK activator group: U2OS cells were added with $1 \mathrm{mM}$ melatonin and $50 \mu \mathrm{M}$ ROCK activator (pentanoic acid); (4) MT + ROCK inhibitor group: U2OS cells were added with $1 \mathrm{mM}$ melatonin and $50 \mu \mathrm{M}$ ROCK inhibitor (ROCK inhibitor-2); (5) Mg-PCL group: U2OS cells were cocultured with Mg-PCL scaffold; (6) $\mathrm{Mg}-\mathrm{PCL}+\mathrm{MT}$ group: U2OS cells were co-cultured with Mg-PCL scaffold loaded with melatonin; (7) Mg-PCL + MT + ROCK activator group: U2OS cells were co-cultured with $\mathrm{Mg}-$ PCL scaffold loaded with melatonin and added with $50 \mu \mathrm{M}$ ROCK activator (pentanoic acid); and (8) $\mathrm{Mg}-$ $\mathrm{PCL}+\mathrm{MT}+\mathrm{ROCK}$ activator group: U2OS cells were 
co-cultured with Mg-PCL scaffold loaded with melatonin and added with $50 \mu \mathrm{M}$ ROCK inhibitor (ROCK inhibitor-2).

\section{Transmission electron microscopy (TEM)}

The cells were scrapped after treatment, collected by centrifugation, washed with PBS and fixed with 5\% glutaraldehyde. After the cells were dehydrated, embedded, sectioned, stained, the mitophagosomes were observed by TEM. The quantity of CIC structures was calculated in 10 fields a view.

\section{Western blot analysis}

Cells or tissues were washed with ice-cold PBS and resuspended in lysis buffer $(50 \mathrm{mM}$ Tris- $\mathrm{HCl}, 150 \mathrm{mM} \mathrm{NaCl}$, $1 \%$ NP- $40,0.5 \%$ sodium deoxycholate, $0.1 \%$ sodium dodecyl sulfate) containing protease and phosphorylase inhibitor cocktails. After centrifugation at $12,000 \mathrm{~g}$ for $30 \mathrm{~min}$ at $4{ }^{\circ} \mathrm{C}$, the supernatant containing total protein was collected and quantified using the BCA protein concentration assay kit (Boster, Hubei, China). The samples were separated by SDS-PAGE and then transferred to polyvinylidene difluoride (PVDF) membranes (Millipore, Massachusetts, USA) (210 mA, between 30 and $120 \mathrm{~min}$ according to the molecular mass of the protein). After blocking for $2 \mathrm{~h}$ with $5 \%$ non-fat milk, the PVDF membranes were incubated with the appropriate primary antibodies (1:500 or $1: 1000$ dilution) at $4{ }^{\circ} \mathrm{C}$ overnight. The blots were then incubated with anti-mouse or antirabbit IgG conjugated to horseradish peroxidase (1:5000 dilution) for $1 \mathrm{~h}$ at room temperature $\left(23-25^{\circ} \mathrm{C}\right)$. Immunoreactive bands were visualized by the EC3 imaging system (UVP Inc., California, USA), and quantified with the Image software (NIH, USA). The ratios between the proteins of interest and loading controls of the same sample were calculated as relative content and expressed graphically. The results were averaged from three independent experiments.

\section{Immunofluorescence}

Frozen sections of human OS tissue were stained with E-cadherin, MT1 and MT2 primary antibodies, followed by Alexa 488-conjugated goat anti-rabbit (Abcam) and Alexa 647-conjugated goat anti-mouse (Abcam) secondary antibodies. Image-Pro Plus 6.0 was used for the quantitative analysis of protein expression, and a mean optical density value [integrated optical density (IOD) divided by the relevant area] was calculated for each visual field.

After treatment, the cells were fixed with $4 \%$ paraformaldehyde at room temperature $\left(23-25{ }^{\circ} \mathrm{C}\right)$ for 15 min. After PBS washing, cells were permeabilized with $0.2 \%$ Triton X-100 for 5 min. Sections were incubated in a blocking buffer containing 5\% BSA for
$30 \mathrm{~min}$ at room temperature $\left(23-25{ }^{\circ} \mathrm{C}\right)$, followed by incubation with primary antibodies overnight at $4{ }^{\circ} \mathrm{C}$. Secondary antibodies labeled with fluorescein (1:500, Abcam) were applied for $120 \mathrm{~min}$. After incubating with $0.1 \%$ DAPI for $10 \mathrm{~min}$ and another wash with PBS, images were captured on a wide field fluorescent microscope (Olympus, Japan). Each experiment was repeated three times (total, $\mathrm{n}=90$ cells) and the fluorescence intensities were quantified with the Image software. Cells were grown in confocal dishes (Nest, California, USA) and incubated with Mitotracker Red CMXRos (M7512, Invitrogen) for 30 min to mark the mitochondria according to the manufacturer's protocol. The cells were then washed with ice-cold PBS, and analyzed under a confocal laser scanning microscope (Olympus, Tokyo, Japan). Data were averaged from three independent experiments (total, $\mathrm{n}=90$ cells). Image-Pro Plus 6.0 (Media Cybernetics) was used for quantifying mitochondria.

\section{Cell invasion and migration assays}

To examine the effects of melatonin and $\mathrm{CIC}$ formation on the invasiveness of U2OS cells in vitro, we employed a modified Boyden chamber invasion assay with Matrigel coating. After treatment, the cells were seeded into the upper section of the Boyden chamber (Neuro Probe, Cabin John, MD, USA) at densities of $2.0 \times 10^{5} / \mathrm{ml}$, and then incubated for $24 \mathrm{~h}$ at $37^{\circ} \mathrm{C}$. Finally, the cells were counted under a light microscope. Cell migration was measured by a cell invasion assay without Matrigel coating.

\section{Wound-healing assay}

The U2OS cells were plated at $8.5 \times 10^{5} /$ well on 6 -well plates for $48 \mathrm{~h}$ and were wounded by scratching with a pipette tip. They were subsequently incubated with DMEM containing 0.5\% FBS. After repeated treatment at $0,12,24$ and $48 \mathrm{~h}$, the cells were photographed using a phase contrast microscope (Olympus, Japan).

\section{Adenosine triphosphate (ATP) and oxygen consumption measurement}

Mitochondria were separated by the Cell Mitochondria Isolation Kit (Beyotime, Shanghai, China). Mitochondrial ATP levels were measured by the ATP Assay Kit (Sigma, MAK 190); while endogenous basal oxygen consumption was measured using Clark-type electrodes of the Oxygen Consumption Rate Assay Kit (Cayman, 600800). All procedures were done according to the manufacturer's protocol. 


\section{DNA isolation and mitochondrial DNA copy number quantification}

Genomic DNA was isolated using the EasyPrep Genomic DNA Extraction Kit (TOOLS) following the manufacturer's instructions. Mitochondrial DNA (mtDNA) copy number was quantified in $10 \mu \mathrm{l} 2 \mathrm{X}$ SYBR Green PCR Master Mix (Roche) containing $5 \mu \mathrm{M}$ forward and reverse primers, with approximately $10 \mathrm{ng}$ DNA. mtDNA levels were assessed using primers against the mitochondrial gene, ND1, with telomerase reverse transcriptase (TERT) serving as a loading control. Gene expression quantification was based on the comparative cycle threshold (CT) $\left(2^{-\triangle \triangle \mathrm{CT}}\right)$ method. The primer sequences were as follows: ND1: forward, 5'-ACCAT TTGCA GACGC CATAA-3'; reverse, 5'-TAAAT TGTTT GGGCT ACGG-3'; TERT: forward, 5'-CTAGC TCATG TGTCA AGACC CTCT-3'; reverse, 5'-GCCAG CACGT TTCTC TCGTT-3'.

\section{Statistical analysis}

The quantitative variables are expressed as mean \pm standard deviation (SD). All data were analyzed using the GraphPad Prism 6.02 software program. Statistical significance between the two groups was analyzed by the Student's t-test. The one-way analysis of variance (ANOVA) was used to compare multiple groups. A P value of $<0.05$ was considered statistically significant.

\section{Results}

\section{Determination of the characteristics of Mg-PCL scaffold}

Mg-PCL composite offers an ideal candidate for bone tissue engineering with lower corrosion resistance and good biocompatibility. We first prepared an $\mathrm{Mg}-\mathrm{PCL}$ scaffold by 3D-printing. The characteristics of Mg-PCL scaffold were determined. The infrared spectroscopy of PCL was not significantly changed after mixing with Mg. TGA analysis suggested that PCL was sublimated at $412{ }^{\circ} \mathrm{C}$ and the sublimation temperature decreased with the increase of $\mathrm{Mg}$ content (Fig. 1A). Mechanical properties of the scaffold were not significant different among different groups, except that the compressive modulus was significantly decreased at 5\% Mg-PCL (Fig. 1B). SEM showed that the Mg content was not much at the surface but distributed primarily in the interior of scaffold (Fig. 1C). In vitro immersion test indicated that the mineralization thickness and corrosion rate of scaffold was gradually increased along with the increasing of $\mathrm{Mg}$ content (Fig. 1D-F). Taking physicochemical properties and immersion test into account, we selected the $5 \% \mathrm{Mg}-$ PCL for further experiment.

\section{Determination of the effects of Mg-PCL scaffold on the tumor metastasis in vivo}

Further, we established an in vivo OS model in rabbit and inserted the Ti-PCL and Mg-PCL implants into the front edge of the tibial plateau. MicroCT scan results of rabbit tibia showed that the formation of callus in the Mg-PCL scaffold group was increased and the static bone microstructure index was the highest in the three groups, while the traditional Ti-PCL stent was the lowest in the three groups (Fig. 2A). HE staining demonstrated that lung metastasis occurred 2 months after surgery in the Mg-PCL scaffold stent group (Fig. 2B). These data indicated the Mg-PCL scaffold could promote bone growth and might be an ideal candidate for bone tissue engineering; however, it can't inhibit tumor metastasis.

\section{Assessment of $\mathrm{CIC}$ in tumor tissues of OS patients}

It is reported that $\mathrm{CIC}$ phenomenon may promote tumor progression in many types of epithelial tumors [34]. However, its role in OS has not been reported yet. Tumor tissue samples from 30 OS patients (20 fields per sample) were stained with $\mathrm{HE}$ and fluorescent-labeled E-cadherin antibody. As shown in Fig. 3A, both HE and immunofluorescence detected CIC phenomenon. These results indicated that CIC occurred in OS tumor tissues, and mainly by tumor cells swallowing other tumor cells.

Melatonin inhibits OS development through acting on MT1 Melatonin was reported to be an anti-tumor agent for OS by acting on MT1 and MT2 [21, 22]. Immunofluorescence analysis showed that both MT1 and MT2 were expressed in the OS tissues (Fig. 3B). MT1 and MT2 protein expressions in different OS cell lines-U2OS, MG63, HOS and 143B were tested through Western blotting with human osteoblasts hFOB1.19 as the control. The expressions of MT1 in all OS tissues and OS cells were significantly higher, while MT2 expression showed no marked difference compared with the control group (Fig. 3C). Among the OS cell lines, the difference in MT1 protein in U2OS cells was the most obvious. Consequently, the U2OS cell line was selected for the rest of the

(See figure on next page.)

Fig. 1 Characteristics of Mg-PCL scafford. A Infrared spectroscopy, X-Ray diffraction and thermogravimetric analyses of Mg-PCL. B Compressive and tensile characteristics of $\mathrm{Mg}-\mathrm{PCL}$. ${ }^{*} \mathrm{P}<0.05$ compared with $\mathrm{PCL}$ group. $\mathbf{C}$ The surface and cross-section images of the Mg-PCL scaffold with different content of $\mathrm{Mg}$ under scanning electron microscopy. $\mathbf{D}$ In vitro immersion test of $\mathrm{Mg}-\mathrm{PCL}$ scaffold with different content of $\mathrm{Mg}$ at 0-day and 30-day. E Corrosion rate of Mg-PCL scaffold with different content of Mg at 0-day and 30-day. ${ }^{*} \mathrm{P}<0.05$. F The surface morphology of Mg-PCL scaffold with different content of Mg was observed by SEM at 30-day 


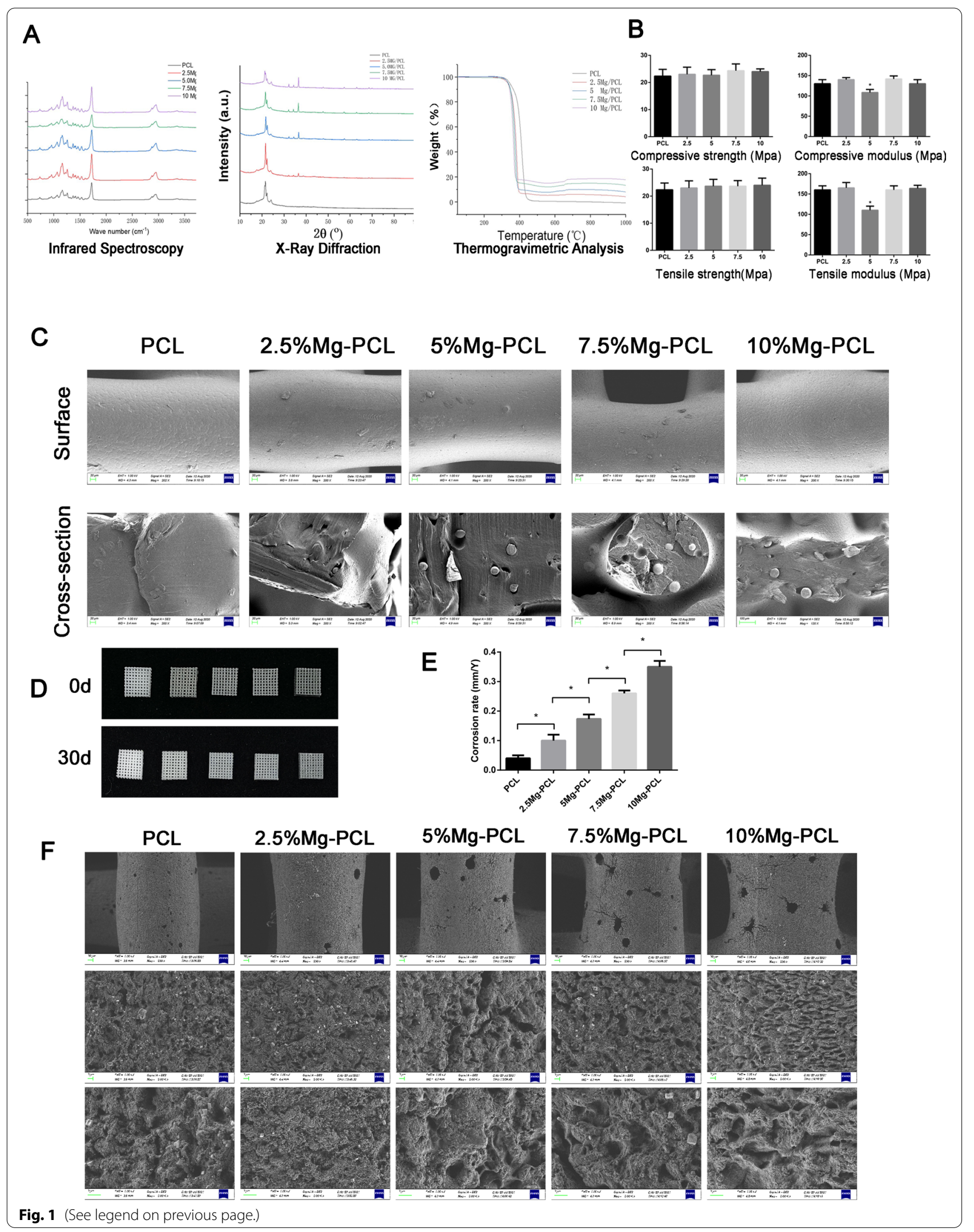




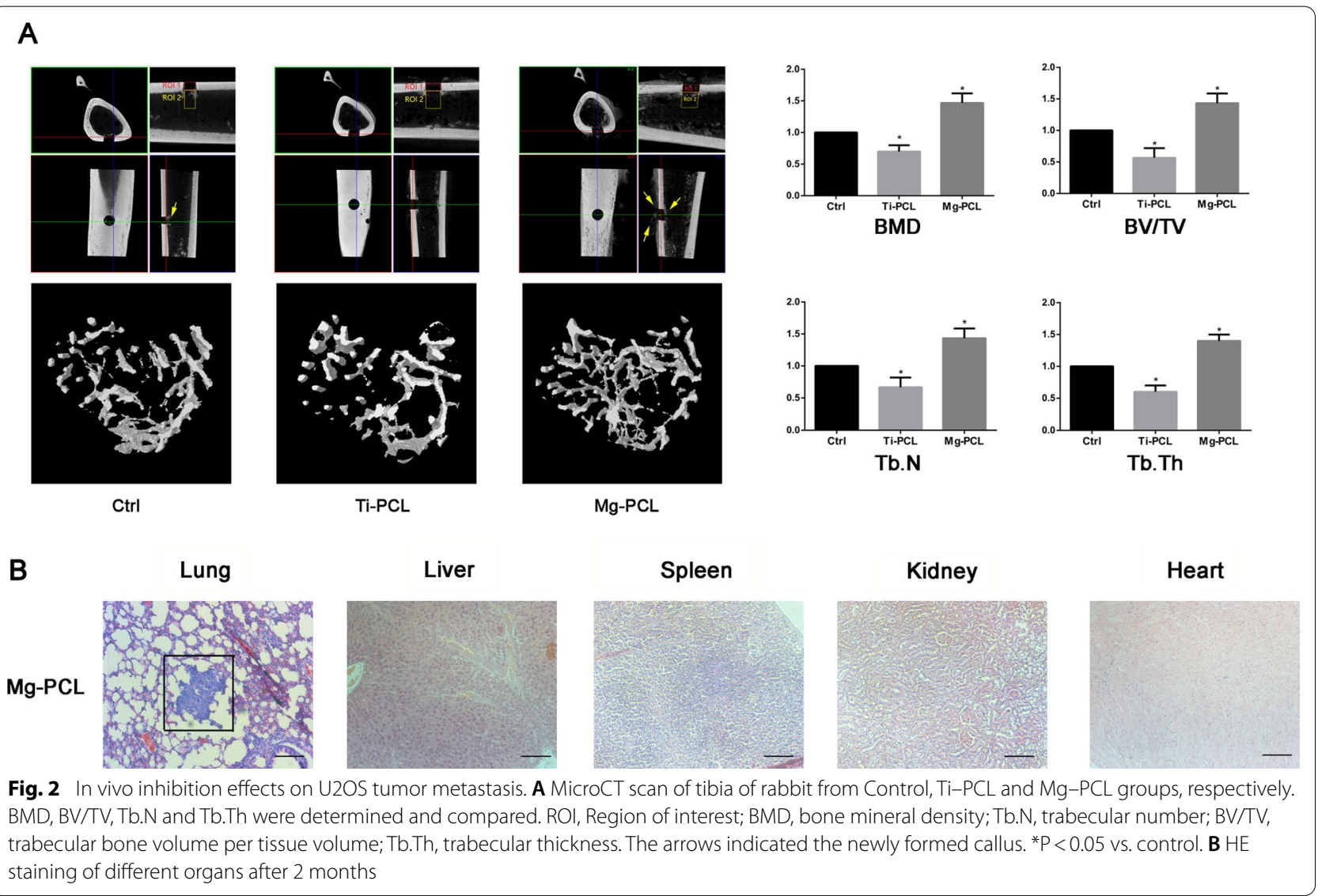

experiment. We also speculated that effects of melatonin on OS cells might be achieved through MT1.

\section{Effect of melatonin on CIC formation and OS cell invasion and migration in vitro}

In order to investigate the effect of melatonin on $\mathrm{CIC}$ phenomenon, U2OS cells were treated with melatonin and $\mathrm{CIC}$ were determined by immunofluorescence analysis and electron microscope. As shown in Fig. 4A and B, the number of CIC structure was significantly reduced with melatonin treatment. It is reported that ROCK proteins positively regulate $\mathrm{CIC}$ formation [38]. In order to investigate the molecular mechanism of melatonin on CIC formation, we treated the U2OS cells with melatonin (MT), MT + MT1 activator (7-Desmethyl-agomelatine) or MT + MT1 inhibitor (S26131) and evaluated the levels of PKA and Rho/ROCK. We found that both $\mathrm{MT}$ and MT + MT1 activator could promote PKA phosphorylation, and reduced Rho and ROCK expressions. In contrast, $\mathrm{MT}+\mathrm{MT} 1$ inhibitor reversed the above results (Fig. 4C). We also found that $\mathrm{MT}+\mathrm{PKA}$ inhibitor reversed the inhibitory effects of MT on Rho and ROCK expressions (Fig. 4D). These results indicated that melatonin may play an important role in inhibiting the Rho/
ROCK signaling pathway of CIC by activating the PKARho/ROCK signaling pathway, thus inhibiting CIC.

Effect of melatonin on mitochondrial physiology of OS cells CIC formation between tumor cells has been reported to alter energy metabolism, which closely related to the occurrence and development of tumors [31, 32]. We treated the U2OS cells with MT, MT + ROCK activator (pentanoic acid) [39], or $\mathrm{MT}+\mathrm{ROCK}$ inhibitor (ROCK inhibitor-2) [40], and determined the relative fluorescence of mitochondria by immunofluorescence. We observed that MT and MT+ROCK inhibitor reduced the relative fluorescence of mitochondria in OS cells $(\mathrm{P}<0.05$, Fig. 5A), downregulated the expression of mitochondrial biogenesis-related proteins PGC1 $\alpha$, NRF1 and TFAM, and reduced the expression of mitochondrial biomarkers TOM20, COX-411 and ATP $\operatorname{Syn} \beta$ ( $P<0.05$, Fig. $5 B)$. In contrast, $M T+R O C K$ activator reversed the above results. We also found significantly higher mtDNA levels in the control and the $\mathrm{MT}+\mathrm{ROCK}$ activator groups than in the MT and $\mathrm{MT}+\mathrm{ROCK}$ inhibitor groups $(\mathrm{P}<0.05$, Fig. $5 \mathrm{C})$. ATP production and $\mathrm{O} 2$ consumption were found to be higher in the control and the MT+ROCK inhibitor 
A
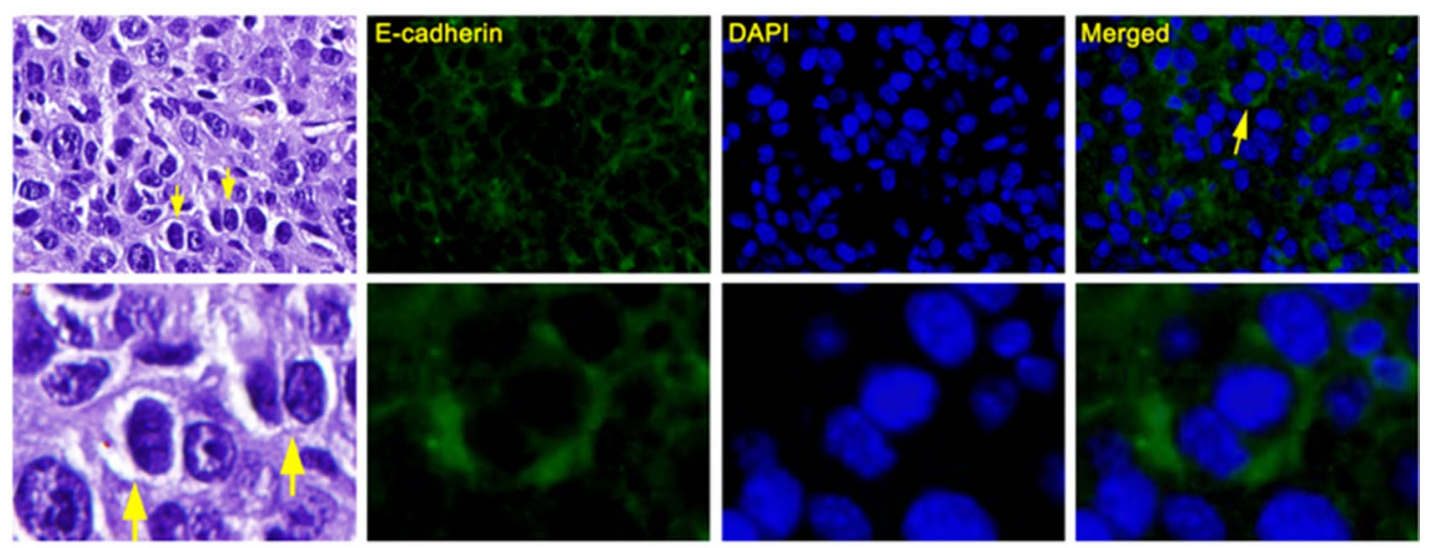

B
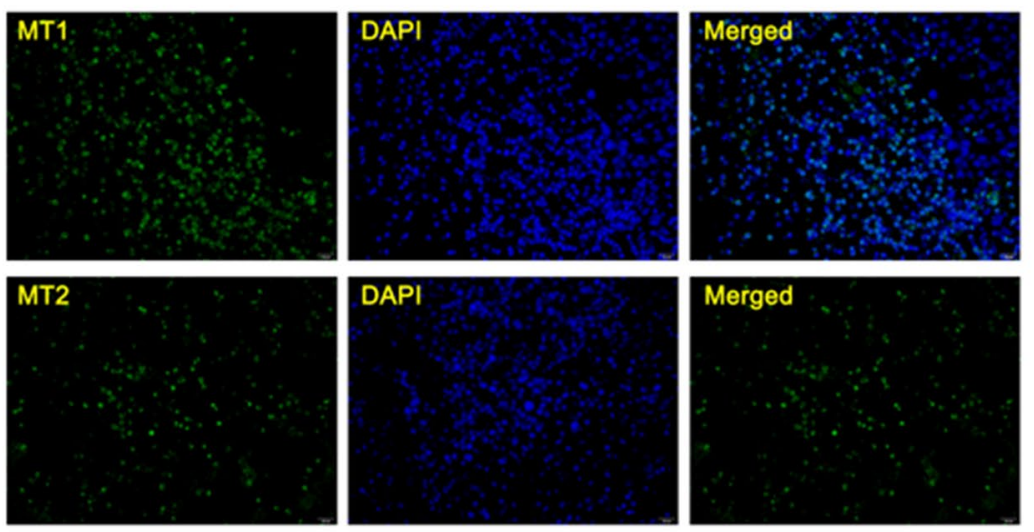

C
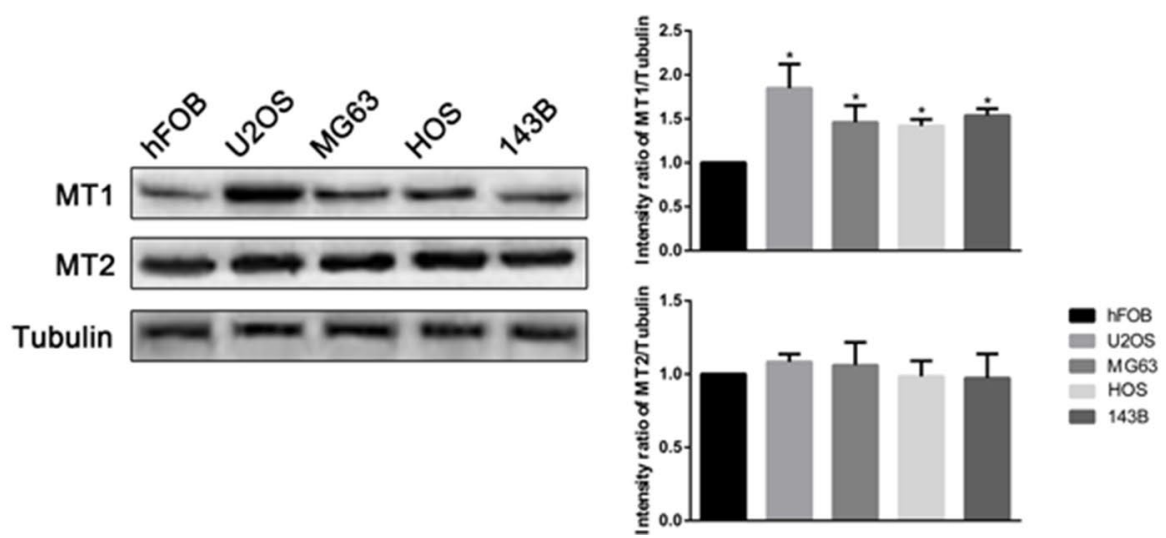

Fig. 3 Cell-in-cell (CIC) structure and expression of melatonin receptor in OS patients and OS cells. A HE and immunofluorescence of CIC in tumor tissue of OS patients. B Immunofluorescence showing MT1 and MT2 expressions in human OS tissues. C Western blot shows MT1 and MT2 expression in $\mathrm{hFOB}, \mathrm{U} 2 \mathrm{OS}, \mathrm{MG} 63, \mathrm{HOS}$ and $143 \mathrm{~B}$ cells. Values represent the mean $\pm \mathrm{SD}$ of three independent experiments. ${ }^{*} \mathrm{P}<0.05 \mathrm{vs}$. control

groups than in the MT and MT+ROCK inhibitor groups (Fig. 5D and E). These findings suggested that melatonin inhibited mitochondrial biogenesis and function in OS cells through the Rho/ROCK-mediated CIC pathway.

\section{Pharmacokinetics and in vivo safety of melatonin}

The pharmacokinetics of melatonin in mice were studied by injecting melatonin into the tail vein at 10,100 and $1000 \mathrm{mg} / \mathrm{kg}$ according to body weight, and collecting blood from the fundus vein to observe the changes 


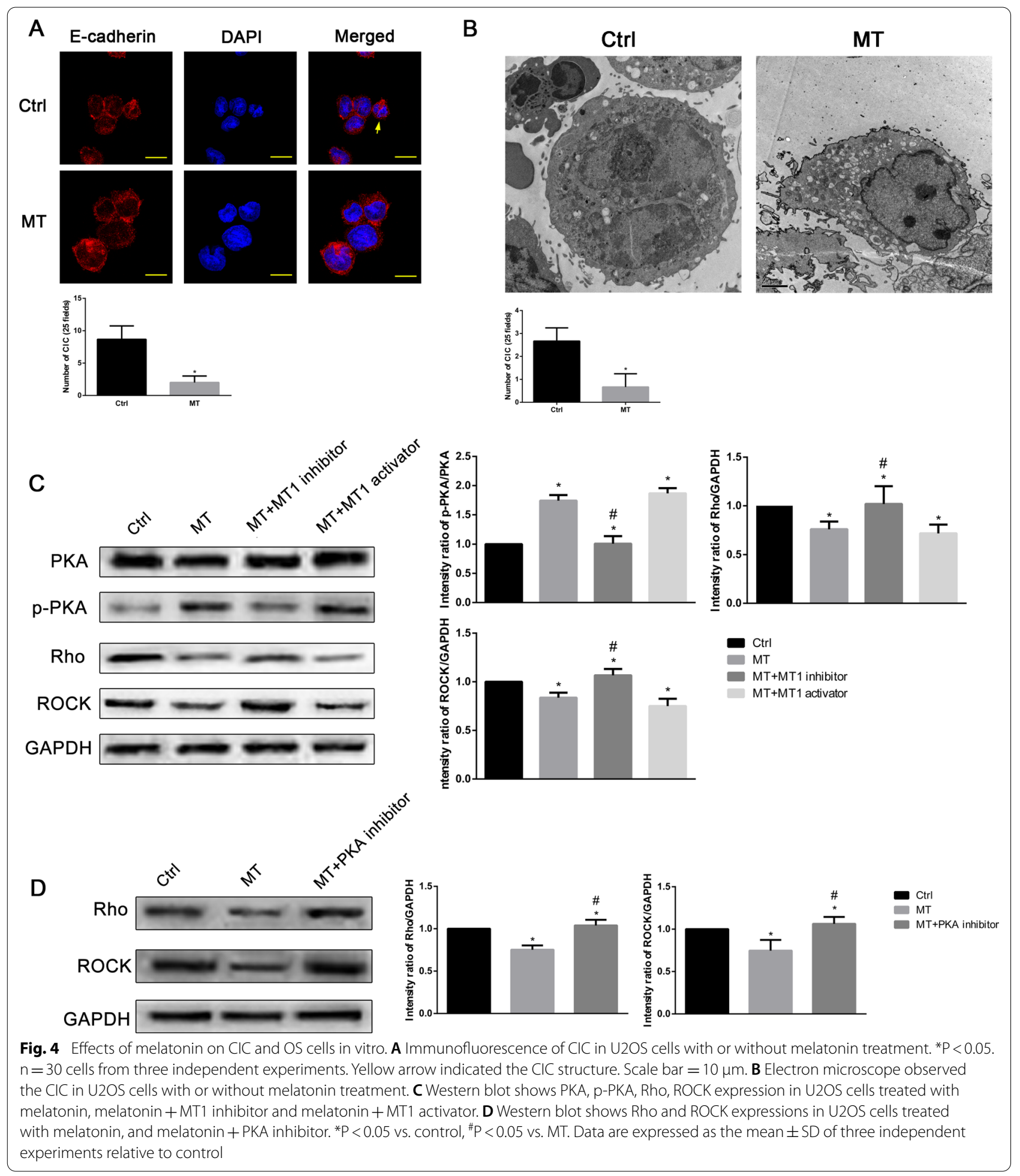

of melatonin concentration in the venous blood. The drug concentrations at different time points are shown in Fig. 6A and Table 2. Melatonin concentration peaked at 30 min after administration maintained with slight decrease for $6 \mathrm{~h}$, and gradually decreased thereafter to near baseline levels at $12 \mathrm{~h}$.

The safety of melatonin in mice was further studied by injecting melatonin into the tail vein of mice once every 
2 days at $3 \mathrm{mg}$ each time. After 30 days, the heart, liver, spleen, lung, kidney and other important organs were collected, sectioned and stained with HE for microscopy observation. The weight of all mice was stable without notable changes (Fig. 6B) and no organ damage was observed in mice treated with melatonin (Fig. 6C). The LE and LC of melatonin loaded SunP Gel G1 at mass concentration of $5 \mathrm{~g} / \mathrm{l}$ are shown in Table 3. Along with the increasing of the mass ratio of melatonin and SunP Gel G1, the LE and LC were increased. The LE reached peak at mass ratio of 1:2 and was decreased at mass ratio of 2:1. The mass ratio of $1: 2$ was selected for further experiments.

The drug release rates of melatonin and melatonin loaded SunP Gel G1 are shown in Fig. 6D and E. The cumulative release rate of melatonin has reached $96.3 \%$ at $10 \mathrm{~h}$, while the drug release cycle of melatonin loaded SunP Gel G1 can reach 14 days and there is no obvious burst release. These results suggest melatonin loaded SunP Gel G1 has ability to control the release of melatonin and effectively achieves the sustained release of melatonin.

\section{Effect of 3D-printed Mg-PCL-MT scaffold on OS progression in vivo}

Since the Mg-PCL scaffold could promote the bone growth and melatonin could inhibit CIC phenomenon, we speculated that melatonin-loaded Mg-PCL scaffold might inhibit OS progression. We found that MT and MT + ROCK inhibitor effectively inhibited the cell proliferation (Fig. 7A) and tumor weight (Fig. 7C) and promoted cell apoptosis (Fig. 7B) of xenograft tumors in nude mice with implanted U2OS cells $(\mathrm{P}<0.05)$, while MT + ROCK activator had no significant effect $(P>0.05)$. These results demonstrated that melatonin inhibited OS growth in vivo and this inhibition could be abolished by ROCK activator. In addition, $\mathrm{Mg}-$ $\mathrm{PCL}+\mathrm{MT}, \mathrm{Mg}-\mathrm{PCL}+\mathrm{MT}+\mathrm{ROCK}$ activator and $\mathrm{Mg}-$ $\mathrm{PCL}+\mathrm{MT}+\mathrm{ROCK}$ inhibitor demonstrated similar effect on cell proliferation with those without Mg-PCL.

HE staining demonstrated that lung metastasis occurred in the control, MT+ROCK activator, $\mathrm{Mg}-$ PCL scaffold and Mg-PCL + MT + ROCK activator groups, while no organ metastasis was observed in other groups (Fig. 8A). We established an in vivo OS model and inserted the Mg-PCL implant and Mg-PCL-MT implant group into the front edge of the tibial plateau. MicroCT scan results of mice tibia showed that the formation of callus in the Mg-PCL implant group and $\mathrm{Mg}-\mathrm{PCL}+\mathrm{MT}$ implant group was increased and the static bone microstructure index was the highest in $\mathrm{Mg}-\mathrm{PCL}+\mathrm{MT}$ implant group (Fig. 8B).

\section{Effect of 3D-printed Mg-PCL-MT scaffold on OS cell invasion and migration in vitro}

The effect of 3D-printed Mg-PCL-MT scaffold on OS cell invasion and migration were assessed by wound-healing assay and Transwell. The wound healing demonstrated that the scratch width was wider in the MT+ROCK inhibitor and the MT groups, but narrower in the MT+ROCK activator group (Fig. 9A). Quantitative analysis suggested that MT and $\mathrm{MT}+\mathrm{ROCK}$ inhibitor groups significantly decreased cell migrated area relative to control $(\mathrm{P}<0.05)$, while $\mathrm{MT}+\mathrm{ROCK}$ activator not $(\mathrm{P}>0.05)$. In addition, $\mathrm{Mg}-$ $\mathrm{PCL}+\mathrm{MT}, \mathrm{Mg}-\mathrm{PCL}+\mathrm{MT}+\mathrm{ROCK}$ activator and $\mathrm{Mg}-$ $\mathrm{PCL}+\mathrm{MT}+\mathrm{ROCK}$ inhibitor demonstrated similar effect on cell migration with those without Mg-PCL (Fig. 9A). Transwell results showed that the number of invaded and migrated cells in the MT and the MT+ROCK inhibitor groups was significantly lower than that in the $\mathrm{MT}+\mathrm{ROCK}$ activator group and the control groups (Fig. 9B). Mg-PCL + MT, Mg-PCL + MT + ROCK activator and $\mathrm{Mg}-\mathrm{PCL}+\mathrm{MT}+\mathrm{ROCK}$ inhibitor demonstrated similar effect on cell migration and invasion with those without Mg-PCL. These results suggested that 3D-printed Mg-PCL-MT scaffold showed similar effects on inhibiting the invasion and migration of OS cells with melatonin.

\section{Discussion}

At present, clinical treatment of OS commonly includes preoperative/neoadjuvant chemotherapy, surgical resection of primary and metastatic tumor areas, and postoperative chemotherapy [41]. Bone defects derived from surgical resection need to be reconstructed using biomaterials. PCL is a biocompatible and biodegradable polymer, however, its biological activity is poor [42]. It is reported that magnesium ion is essential for new bone formation and could induce increase in osteogenic activity $[9,10]$. By $3 \mathrm{D}$ printing technology, we developed an Mg-PCL scaffold and assessed its effects and molecular

\footnotetext{
(See figure on next page.)

Fig. 5 Effects of CIC on mitochondrial physiology of OS cells. U2OS cells were treated with melatonin, melatonin + ROCK activator and melatonin + ROCK inhibitor for $24 \mathrm{~h}$. A Immunofluorescence of mitochondria in U2OS cells. Scale bar $=20 \mu \mathrm{m}$. B Western blot shows TOM20, COX411, ATP syn $\beta$, PGC1a, NRF1 and TFAM expressions. C mtDNA levels of U2OS cells. D Mitochondrial ATP production in U2OS cells. E Mitochondrial $\mathrm{O}_{2}$ consumption in U2OS cells. ${ }^{*} \mathrm{P}<0.05 \mathrm{vs}$. control, ${ }^{\#} \mathrm{P}<0.05 \mathrm{vs}$. MT. Data are expressed as mean $\pm \mathrm{SD}$ fold induction of three independent experiments relative to control
} 


\section{A}
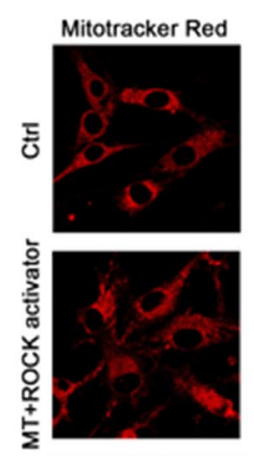

令
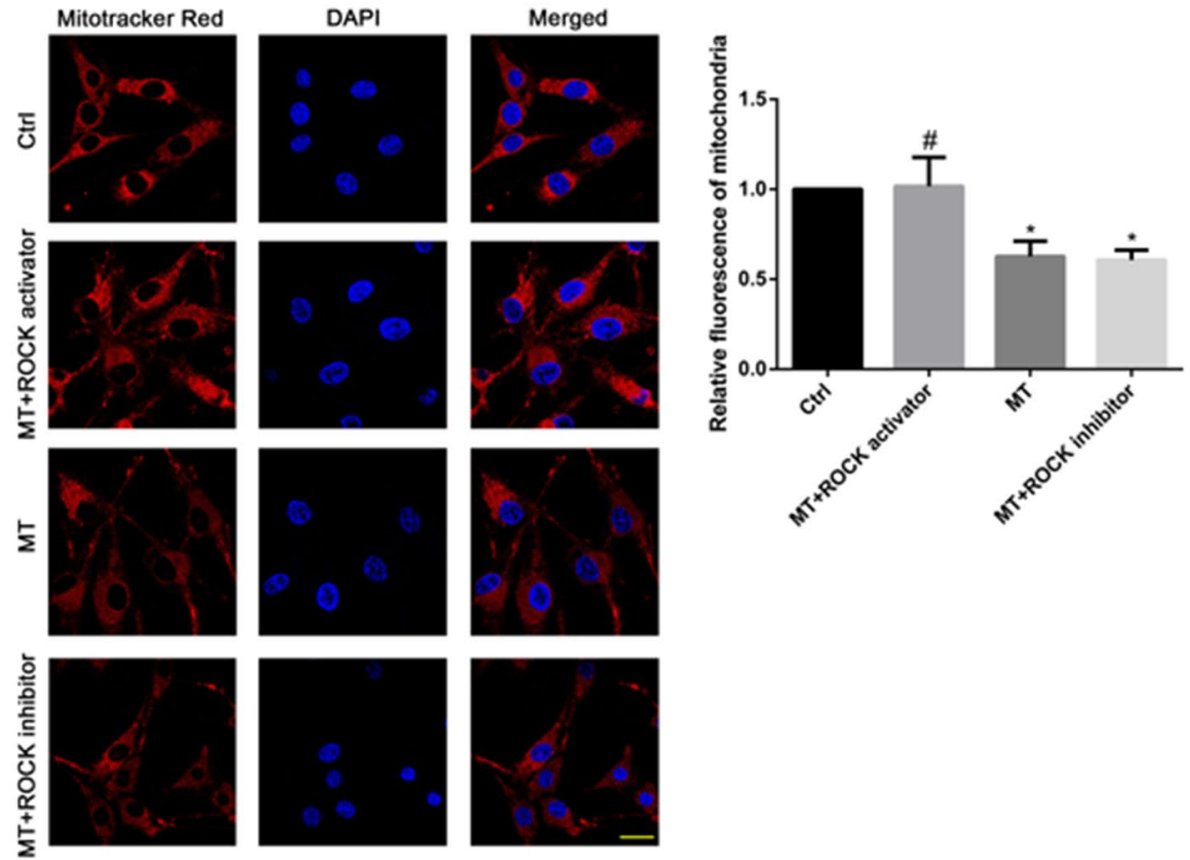

B
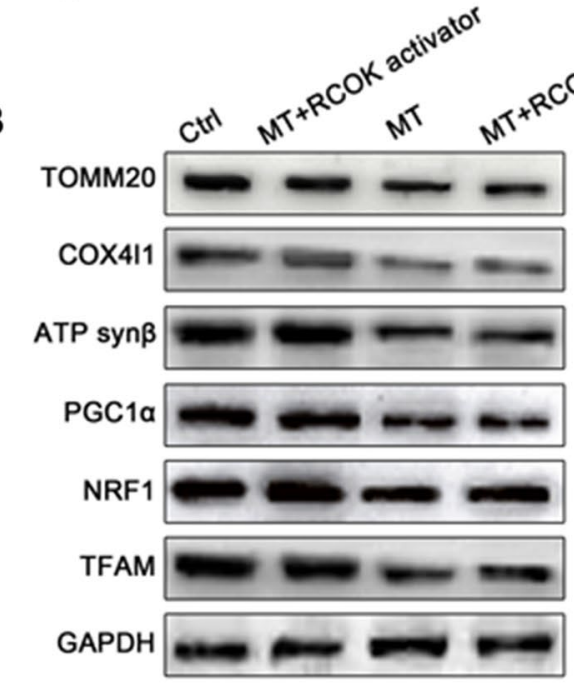

동 1.5
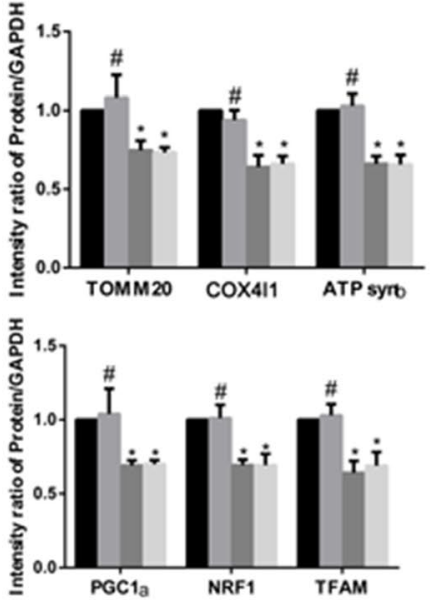

- arr

- $M T+R O C K$ activator

- MT

MT+ROCK intibitor

C

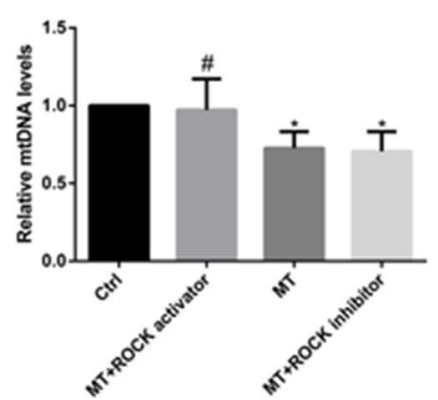

D

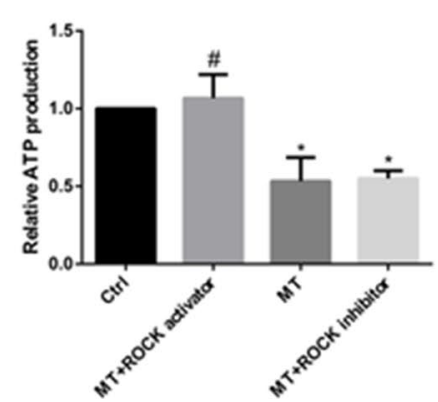

E

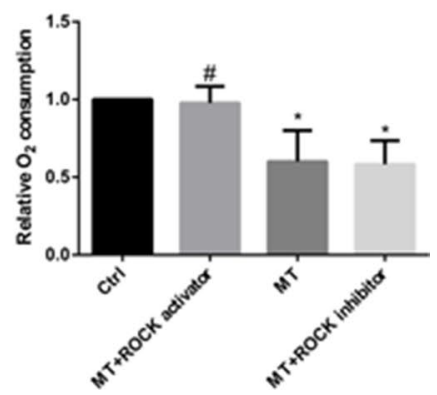

Fig. 5 (See legend on previous page.) 
A

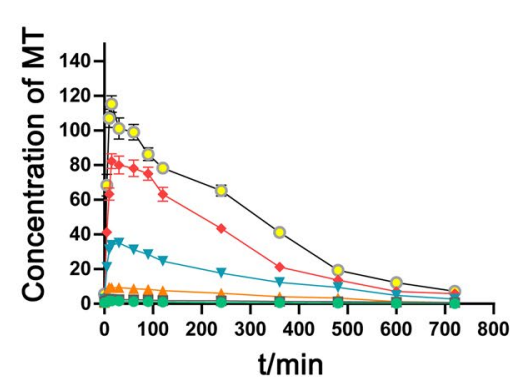

C
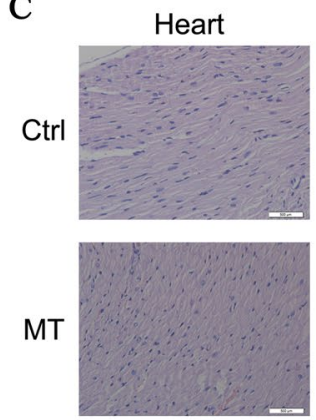

D

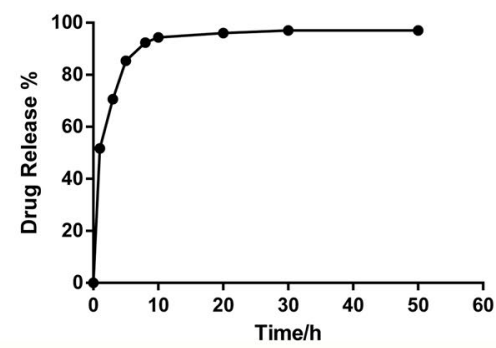

Liver

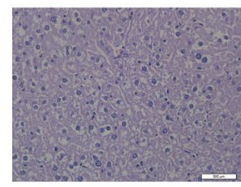

$\rightarrow$ Ctrl+LM

- OS+LM

- Ctrl+MM

$\rightarrow$ OS+MM

$\rightarrow \mathrm{Ctrl}+\mathrm{HM}$

$-\infty-\mathrm{OS}+\mathrm{HM}$
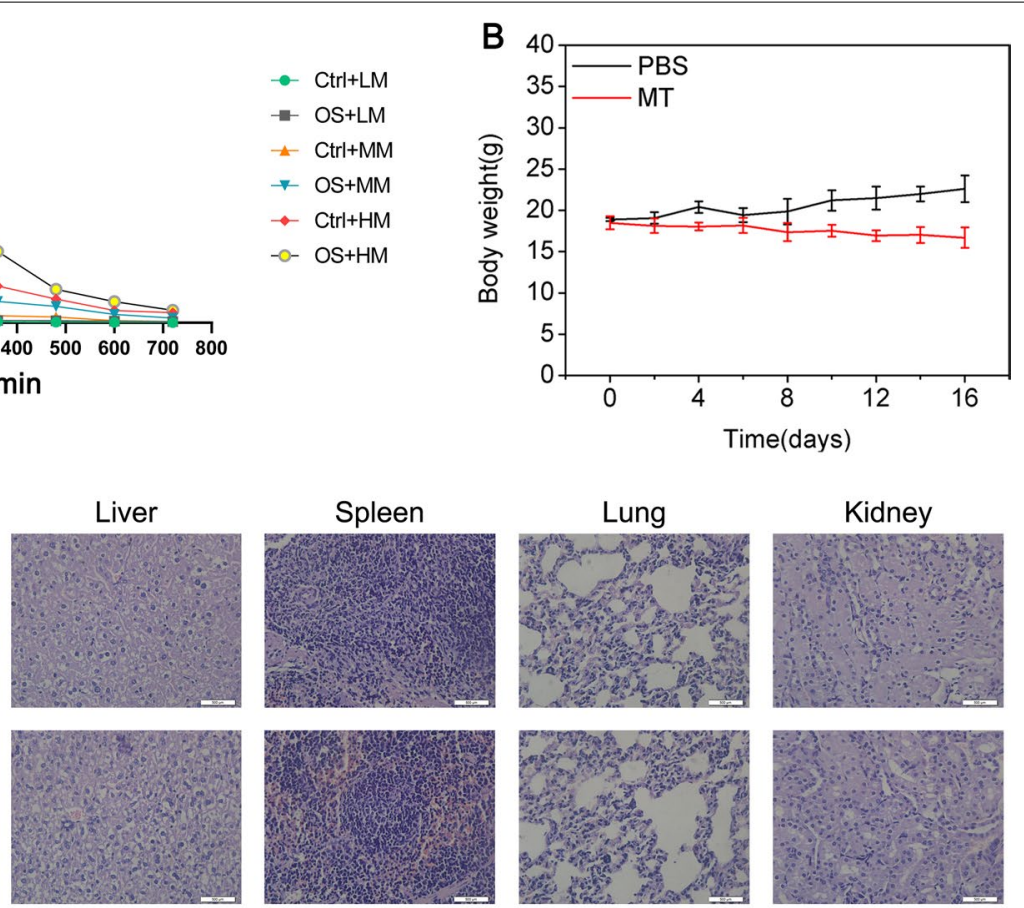

E

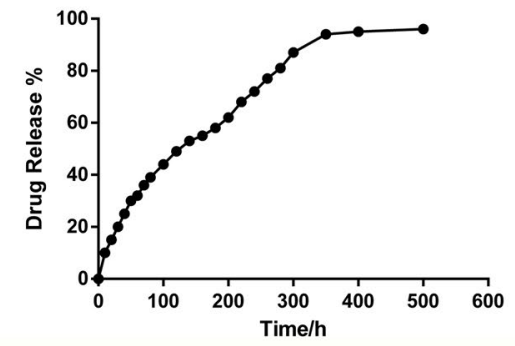

Fig. 6 Pharmacokinetics and in vivo safety of melatonin. A Concentration of melatonin at different time points of mice with or without tumor. B Changes of body weight. C Ex vivo histological analyses of main organ sections from U2OS osteosarcoma-xenografted mice after melatonin treatment. D The drug release rate of melatonin. E The drug release rate of melatonin loaded SunP Gel G1

mechanism on inhibiting OS growth and metastasis. Results demonstrated that Mg-PCL scaffold could promote bone growth and might be an ideal candidate for bone tissue engineering; however, it can't inhibit tumor metastasis. This result is partly consistent with the study of Zhao et al., which demonstrated PCL/10\% Mg composite scaffolds could promote bone defect repair at an early stage with good cytocompatibility [43].

OS cells are not sensitive to radiotherapy [44, 45], and the existing chemotherapeutic anti-tumor drugs are limited by poor tumor specificity which often results in systemic toxicity. In addition, long-term use of chemotherapy drugs can lead to drug resistance as well as decrease in life quality of patients $[46,47]$. Melatonin, as a hormone naturally produced in the human body, has the natural advantages of good biocompatibility and high safety. In this study, the safety of melatonin was proven by HE staining and pharmacokinetics analyses in the liver, lung and kidney of mice. However, the half-life of melatonin is short, which hampers its clinical use in medical materials. Drug delivery system was investigated to settle this problem. For example, Altindal et al. [48] prepared a sustained melatonin release system to inhibit OS proliferation. In this study, we used hydrogel to load melatonin on Mg-PCL scaffold. The hydrogel can construct a good biologically active interface on the surface of the Mg-PCL scaffold, and has better biocompatibility [49]. Our results suggested the cumulative release rate of melatonin has reached $96.3 \%$ at $10 \mathrm{~h}$, while the drug release cycle of melatonin loaded SunP Gel G1 can reach 14 days and there is no obvious burst release. These results 
Table 2 The concentration of melatonin in the plasma of mice

\begin{tabular}{|c|c|c|c|c|c|c|c|c|c|}
\hline \multirow[t]{2}{*}{$t / m i n$} & \multicolumn{3}{|l|}{ LM } & \multicolumn{3}{|l|}{ MM } & \multicolumn{3}{|l|}{ HM } \\
\hline & Ctrl & os & $\mathbf{P}$ & Ctrl & os & $\mathbf{P}$ & Ctrl & os & $\mathbf{P}$ \\
\hline 0 & $0.514 \pm 0.075$ & $1.024 \pm 0.112$ & $<0.05$ & $0.671 \pm 0.815$ & $2.631 \pm 0.916$ & $<0.05$ & $3.751 \pm 0.975$ & $5.543 \pm 0.893$ & $<0.05$ \\
\hline 5 & $0.853 \pm 0.115$ & $1.873 \pm 0.303$ & $<0.05$ & $5.034 \pm 1.326$ & $21.026 \pm 2.116$ & $<0.05$ & $41.267 \pm 1.715$ & $68.375 \pm 6.326$ & $<0.05$ \\
\hline 10 & $1.602 \pm 0.157$ & $2.724 \pm 0.312$ & $<0.05$ & $9.423 \pm 1.723$ & $31.317 \pm 1.893$ & $<0.05$ & $63.365 \pm 3.612$ & $107.067 \pm 5.215$ & $<0.05$ \\
\hline 15 & $1.531 \pm 0.142$ & $2.513 \pm 0.287$ & $<0.05$ & $9.324 \pm 1.825$ & $33.861 \pm 2.004$ & $<0.05$ & $82.326 \pm 4.267$ & $115.335 \pm 4.756$ & $<0.05$ \\
\hline 30 & $1.501 \pm 0.145$ & $2.471 \pm 0.275$ & $<0.05$ & $9.253 \pm 1.741$ & $35.034 \pm 1.246$ & $<0.05$ & $80.034 \pm 5.109$ & $101.231 \pm 6.216$ & $<0.05$ \\
\hline 60 & $1.234 \pm 0.092$ & $2.364 \pm 0.259$ & $<0.05$ & $8.732 \pm 1.626$ & $31.125 \pm 0.896$ & $<0.05$ & $78.131 \pm 4.756$ & $99.034 \pm 4.489$ & $<0.05$ \\
\hline 90 & $1.174 \pm 0.081$ & $2.157 \pm 0.221$ & $<0.05$ & $8.328 \pm 1.273$ & $28.471 \pm 0.791$ & $<0.05$ & $75.022 \pm 3.792$ & $86.328 \pm 3.681$ & $<0.05$ \\
\hline 120 & $0.911 \pm 0.067$ & $1.873 \pm 0.203$ & $<0.05$ & $7.519 \pm 0.935$ & $24.532 \pm 0.687$ & $<0.05$ & $63.237 \pm 4.102$ & $78.265 \pm 2.436$ & $<0.05$ \\
\hline 240 & $0.734 \pm 0.045$ & $1.684 \pm 0.197$ & $<0.05$ & $6.051 \pm 0.587$ & $17.672 \pm 0.836$ & $<0.05$ & $43.414 \pm 2.891$ & $65.354 \pm 3.196$ & $<0.05$ \\
\hline 360 & $0.551 \pm 0.031$ & $1.341 \pm 0.164$ & $<0.05$ & $4.034 \pm 0.435$ & $12.336 \pm 0.578$ & $<0.05$ & $21.243 \pm 1.946$ & $41.246 \pm 2.262$ & $<0.05$ \\
\hline 480 & $0.374 \pm 0.025$ & $1.216 \pm 0.137$ & $<0.05$ & $3.276 \pm 0.375$ & $9.477 \pm 0.433$ & $<0.05$ & $13.635 \pm 2.121$ & $19.348 \pm 1.757$ & $<0.05$ \\
\hline 600 & $0.238 \pm 0.023$ & $0.875 \pm 0.125$ & $<0.05$ & $1.216 \pm 0.203$ & $4.827 \pm 0.327$ & $<0.05$ & $7.044 \pm 1.128$ & $12.134 \pm 1.113$ & $<0.05$ \\
\hline 720 & $0.124 \pm 0.017$ & $0.649 \pm 0.116$ & $<0.05$ & $0.671 \pm 0.194$ & $2.715 \pm 0.366$ & $<0.05$ & $5.768 \pm 1.142$ & $7.215 \pm 0.575$ & $<0.05$ \\
\hline
\end{tabular}

Statistical significance between the two groups was analyzed by the Student's t-test

$L M$ low concentration of melatonin, $M M$ medium concentration of melatonin, $H M$ high concentration of melatonin, $O S$ osteosarcoma

Table 3 The loading efficiency and capacity of melatoninloaded SunP Gel G1 gel

\begin{tabular}{llllll}
\hline \multicolumn{7}{c}{$\mathbf{m}(\mathbf{M T}): \mathbf{m}($ SunP Gel G1) } \\
\hline & $1: 4$ & $1: 3$ & $1: 2$ & $1: 1$ & $2: 1$ \\
LE (\%) & 67.85 & 76.22 & 85.38 & 79.72 & 77.55 \\
LC (\%) & 13.57 & 19.06 & 28.46 & 39.86 & 51.70 \\
\hline
\end{tabular}

LE loading efficiency, $L C$ loading capacity, $m$ mass

suggested that the hydrogel can slow down the release of melatonin to achieve the effect of sustained release. In addition, through in vivo and in vitro experiments, the exact therapeutic effects of melatonin on OS were confirmed. These results demonstrated that melatonin is an effective and valuable treatment option for OS. In the result of MicroCT scan, it is showed that the formation of callus in the Mg-PCL implant group and $\mathrm{Mg}-\mathrm{PCL}+\mathrm{MT}$ implant group was increased and the static bone microstructure index was the highest in $\mathrm{Mg}-\mathrm{PCL}+\mathrm{MT}$ implant group. There have been several studies on the treatment of OS with melatonin. Qu et al. [2] and Liu et al. [50] showed that melatonin inhibited the proliferation and migration of OS cells. Maria et al. and Balci Yuce et al. demonstrated that melatonin could regulate bone formation, growth and differentiation as well as exert anti-osteoporosis effects [23,
24]. Zhang et al. demonstrated that melatonin may reduce the level of autophagy in osteoblasts and delay diabetesinduced osteoporosis by inhibiting the ERK signaling pathway [51]. However, the underlying mechanism via which melatonin inhibits OS progression has not been systematically studied or elucidated.

Unlike conventional chemotherapeutic drugs which act by poisoning tumor cells or activating tumor immunity, treatment targeted towards the CIC phenomenon in OS tissues would suppress the highly energy-consuming nature of OS. As a highly specific treatment, this would have no side effects on normal tissue cells and would effectively inhibit OS development while exerting the lowest cost in terms of tissue damage. In this study, CICs were found in clinical samples of OS patients. With the development of research on the CIC phenomenon, its biological significance is gradually becoming clear. Overholtzer et al. has summarized the possible biological roles of CIC [29], and described that they largely depended on the types of interacting cells. Therefore, we further studied the effects of melatonin on the CIC phenomenon, and elucidated the molecular biological mechanisms connecting melatonin and OS treatment.

The relationship between melatonin and CIC has not been reported, but the relationship between Rho/ROCK

(See figure on next page.)

Fig. 7 Effect of 3D-printed Mg-PCL-MT scaffold on OS in vivo. A immunofluorescence and quantitative analysis of Ki-67 in MT+ ROCK activator, $M T, M T+R O C K$ inhibitor, Mg-PCL, Mg-PCL + MT, Mg-PCL+MT+ROCK activator and Mg-PCL+MT+ROCK inhibitor groups. * $P<0.05$ vs. control. B TUNEL staining of tumor and quantitative analysis in MT + ROCK activator, MT, MT + ROCK inhibitor, Mg-PCL, Mg-PCL + MT, Mg$\mathrm{PCL}+\mathrm{MT}+\mathrm{ROCK}$ activator and Mg-PCL+MT+ROCK inhibitor groups. ${ }^{*} \mathrm{P}<0.05 \mathrm{vs}$. control. C Represent photographs and tumor weight curves of U2OS tumors in MT + ROCK activator, MT, MT + ROCK inhibitor, Mg-PCL, Mg-PCL + MT, Mg-PCL + MT + ROCK activator and Mg-PCL + MT + ROCK inhibitor groups. Values are presented as the mean $\pm S D$ of three independent experiments. ${ }^{*} P<0.05$ vs. control 
A

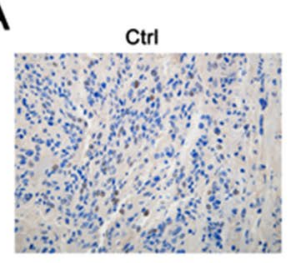

$\mathrm{Mg}-\mathrm{PCL}$

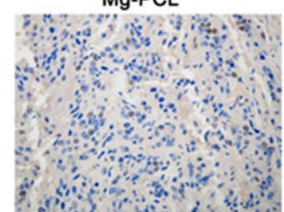

की

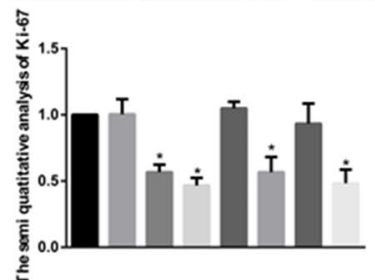

B
MT+ROCK activator

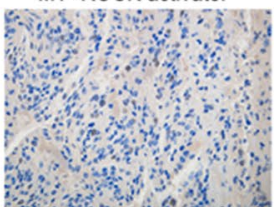

$\mathrm{Mg}-\mathrm{PCL}+\mathrm{MT}$

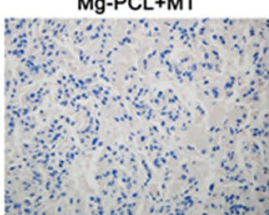

- ${ }_{M T+R}$

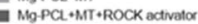

MgPCL+MT•ROCK inhbitor

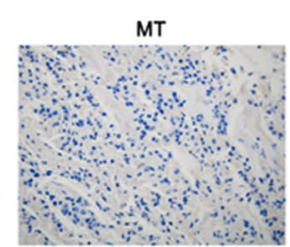

MT+ROCK inhibitor

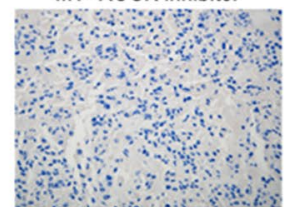

$\mathrm{Mg}-\mathrm{PCL}+\mathrm{MT}+\mathrm{ROCK}$ activato

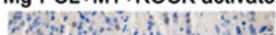

$M g-P C L+M T+R O C K$ inhibitor
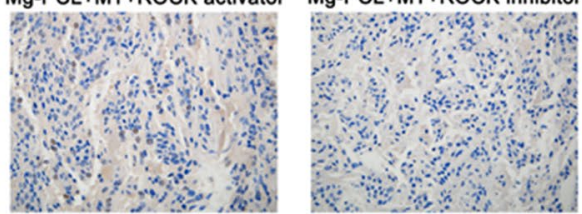
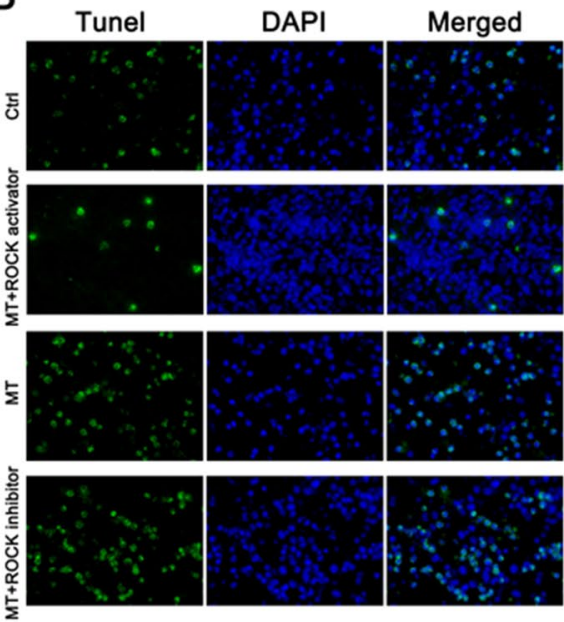

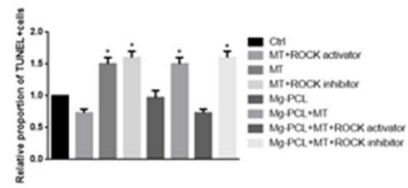

\section{DAP}
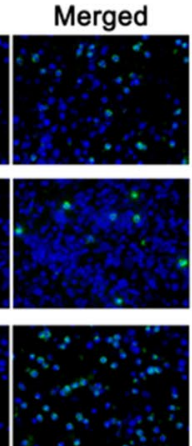

C

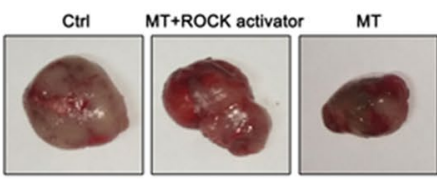

$\mathrm{Mg} \cdot \mathrm{PCL}$

$\mathrm{Mg}-\mathrm{PCL}+\mathrm{MT}$ + ROCK activator

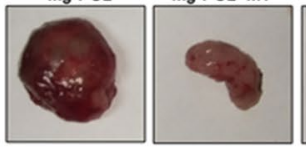

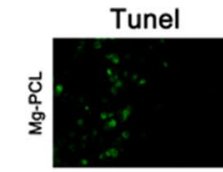

DAPI

Merged
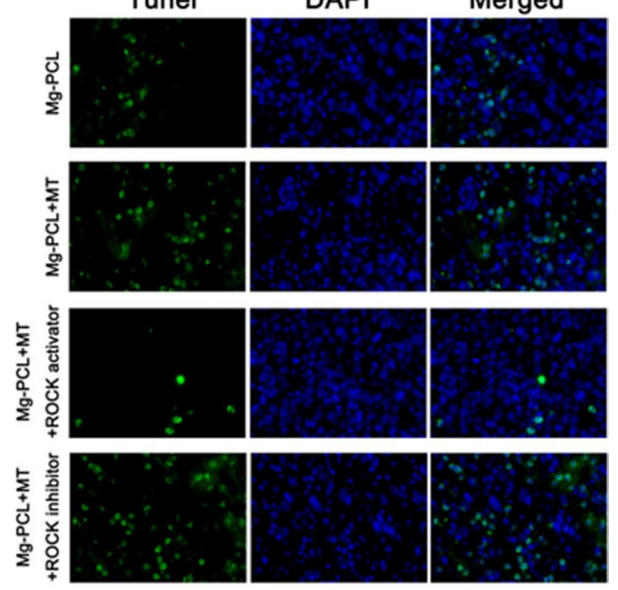

Fig. 7 (See legend on previous page.)

$\mathrm{Mg} \cdot \mathrm{PCL}+\mathrm{M}$
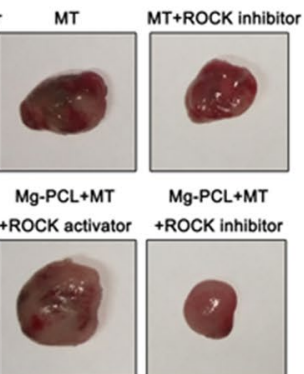

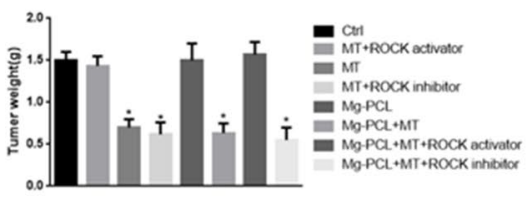



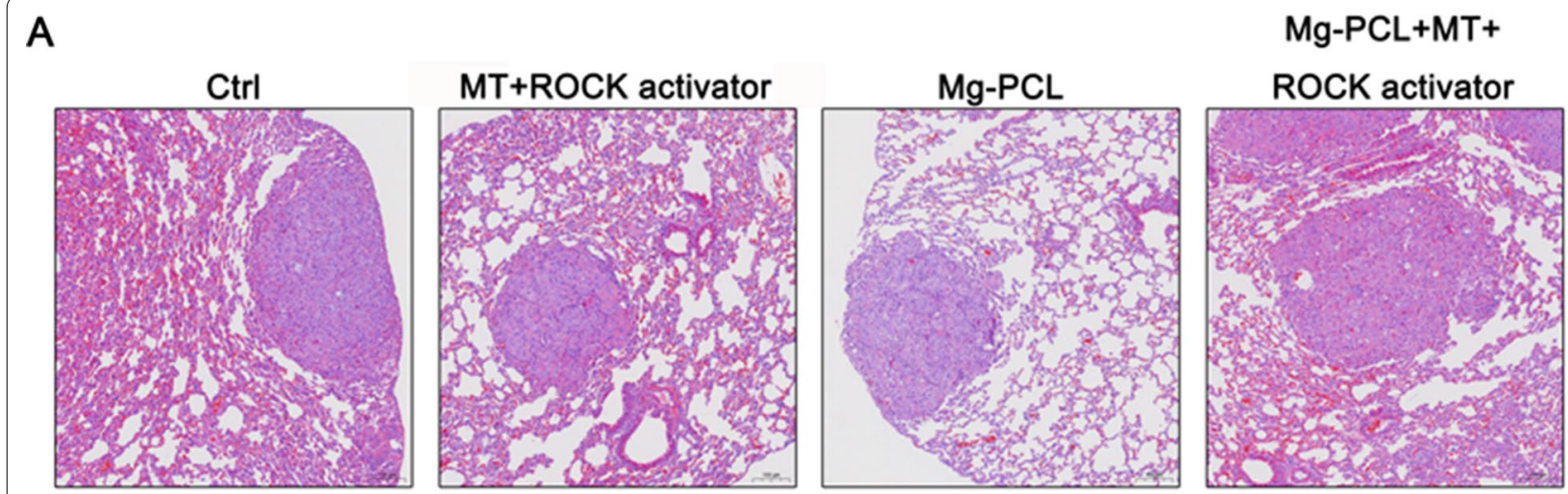

B
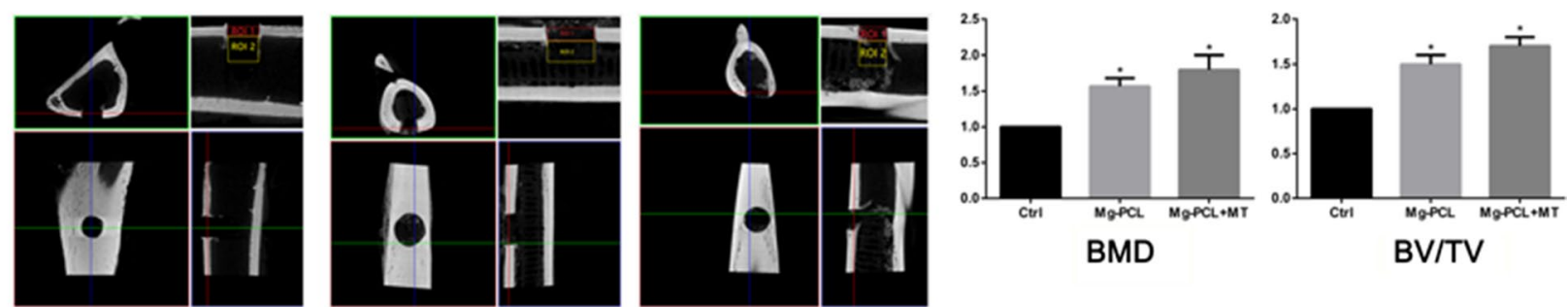

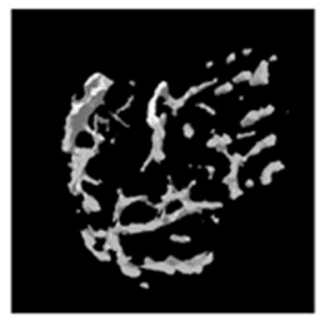

Ctrl

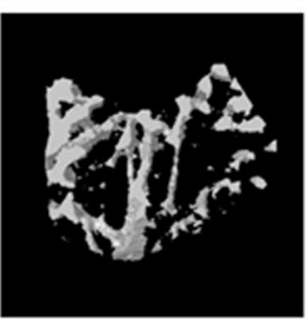

Mg-PCL

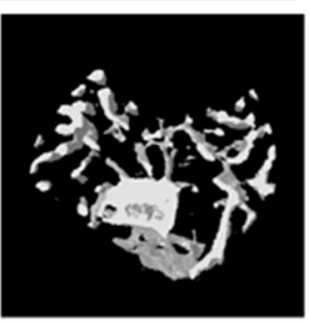

$\mathrm{Mg}-\mathrm{PCL}+\mathrm{MT}$

Fig. 8 Effect of 3D-printed Mg-PCL-MT scaffold on the bone growth in vivo. A HE staining of tumor sections. B MicroCT scan of Control, Mg-PCL and Mg-PCL + MT. BMD, BV/TV, Tb.N and Tb.Th were determined and compared. The arrows indicated the newly formed callus. ${ }^{*} \mathrm{P}<0.05$ vs. control

and $\mathrm{CIC}$ formation has been identified [38]. The effect of melatonin on cAMP/PKA -Rho/ROCK signaling pathway were determined. We found MT1 activator could activate the cAMP/PKA signaling pathway, which was consistent with the report of Schuster et al. [52], and also confirmed a regulatory relationship between PKA and Rho [53]. Therefore, we proposed that melatonin can activate the cAMP/PKA pathway through its receptor, and consequently inhibit the Rho/ROCK pathway, thus interfering with the formation of CIC. Our hypothesis was confirmed, and we successfully identified the regulatory relationship between melatonin and $\mathrm{CIC}$ for the first time.

CIC formation between tumor cells has been reported to alter energy metabolism, which closely related to the occurrence and development of tumors [31, 32]. Mitochondria are organelles that carry genetic information and energy. Wang et al. demonstrated that mitochondria could be transferred among cells through CIC phenomenon [54]. We therefore confirm that CIC affects OS cell

\footnotetext{
(See figure on next page.)

Fig. 9 Effect of 3D-printed Mg-PCL-MT scaffold on OS cell invasion and migration in vitro. A Wound healing results and quantitative analysis of U2OS cells under MT + ROCK activator, MT, and MT + ROCK inhibitor, Mg-PCL, Mg-PCL + MT, Mg-PCL + MT + ROCK activator and Mg$\mathrm{PCL}+\mathrm{MT}+\mathrm{ROCK}$ inhibitor treatments at 0, $12 \mathrm{~h}, 24$ and $48 \mathrm{~h}$. Scale bar $=200 \mu \mathrm{m}$. B Transwell assay of the U2OS cells under MT + ROCK activator, MT, and MT + ROCK inhibitor, Mg-PCL, Mg-PCL + MT, Mg-PCL + MT + ROCK activator and Mg-PCL + MT + ROCK inhibitor treatments for 24 h. Scale bar $=50 \mu \mathrm{m}$. Values represent the mean \pm SD of three independent experiments. ${ }^{*} P<0.05$ vs. control
} 


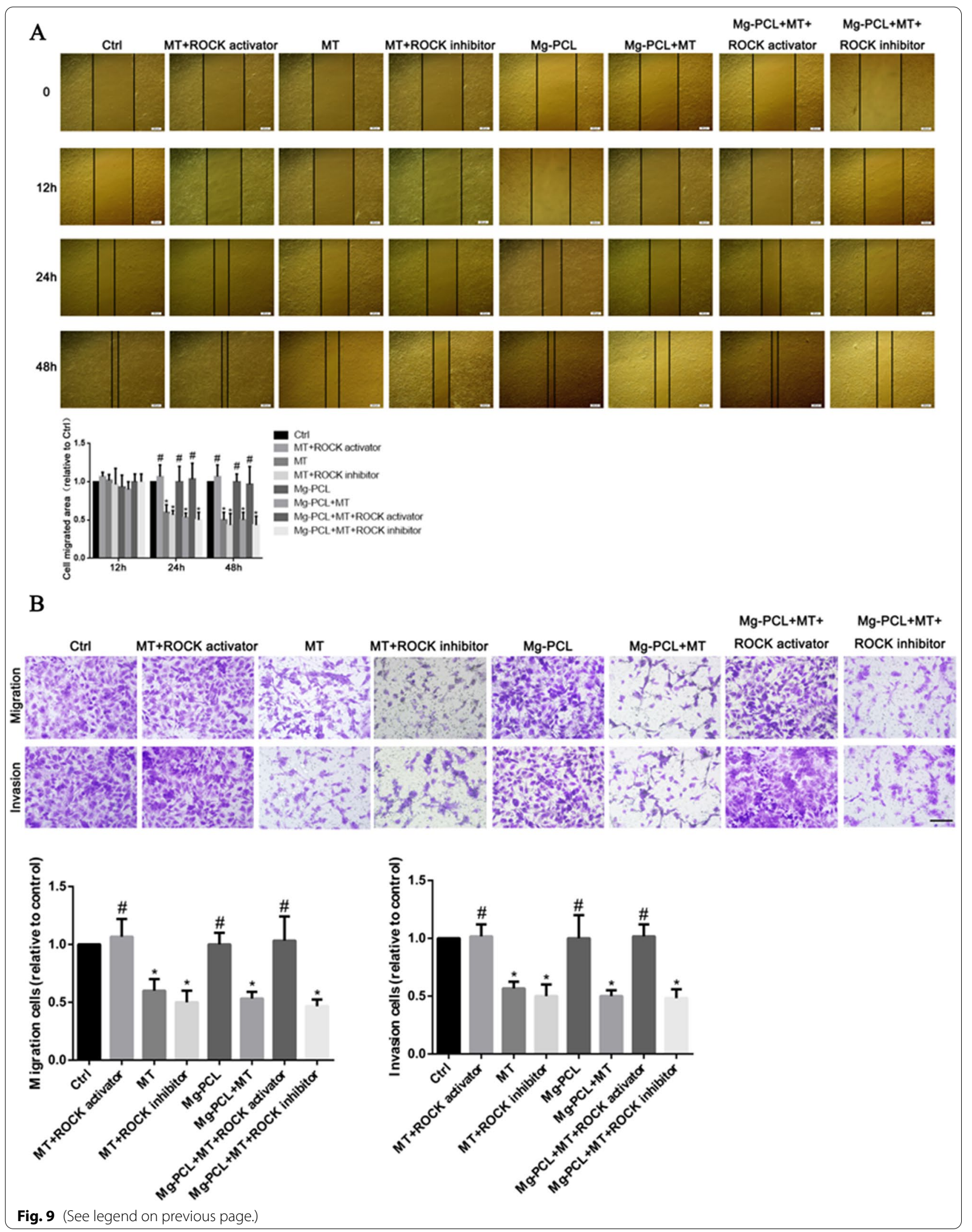


function by interfering with its energy metabolism. Previous studies have revealed that under extreme conditions such as a nutrient-deficient microenvironment, host cells adapted by swallowing effector cells. Leibman et al. after observing the degradation of lymphocytes in host oocytes, suggested that degraded lymphocytes may provide nutrition for host cells [55]. Trowell et al. proposed a similar hypothesis, and observed lymphocyte degradation in fibroblasts and thymic reticulum cells in vitro [56]. Ioachim have demonstrated lymphocyte degradation in a variety of cells in vitro $[29,57]$, while Fais et al. found that metastatic melanoma cells could survive by ingesting live $\mathrm{T}$ cells in the absence of nutrients $[31,32,58]$. In this study, we showed that melatonin inhibited mitochondrial biogenesis and function in OS cells through the Rho/ ROCK-mediated CIC pathway.

In the present study, we found that MT1 expression was upregulated in OS cells. We demonstrate that Mg-PCL-MT could benefit the tumor suppression of melatonin, and our novel melatonin-loaded Mg-PCL scaffolds synergistically inhibited the key CIC pathway, Rho/ROCK, through the cAMP/PKA signaling pathway, interfering with the mitochondrial physiology of OS cells, and thus exerted an anti-invasion and anti-metastasis effect on OS cells. Our results, for the first time, lay foundation for developing melatonin-loaded Mg-PCL scaffolds into a new local adjuvant therapy for OS. Despite having systematically evaluated the molecular biological mechanisms of melatonin in the CIC phenomenon and in OS progression, the underlying mechanism of MT1 expression in OS cells, as well as the epigenetics and proteomics of patient samples, needs to be further explored.

\section{Acknowledgements}

None.

\section{Authors' contributions}

WLZ, WZ, and ZYZ designed and conceived the study. DYZ, JXQ, ZYY, and $Z H C$ performed the in vitro and in vivo experiments. WLZ and WZ drafted the manuscript. XJZ and XLZ provided suggestions and revised the manuscript. WLZ and WZ contributed equally to this work. All authors read and approved the manuscript.

\section{Funding}

This study was funded by the Science and Technology Program of Liaoning Province (No. 20180551005 and No. 2019-MS-391).

\section{Data availability statement}

The data that support the findings of this study are available on request from the corresponding author.

\section{Declarations}

\section{Ethics approval and consent to participate}

All procedures performed in studies involving human participants were in accordance with the ethical standards of the institutional and national research committee and with the 1964 Helsinki declaration and its later amendments or comparable ethical standards. Written informed consent has been received from all included patients and the research protocol has been approved by the Fourth Affiliated Hospital of China Medical University Ethics Committee (No. EC-2018-KS-058).

\section{Consent for publication}

Written informed consent has been received from all included patients.

\section{Competing interests}

All authors declared they have no conflict of interest.

\section{Author details}

${ }^{1}$ Department of Orthopedics, The Fourth Affiliated Hospital of China Medical University, Shenyang 110032, Liaoning, China. ${ }^{2}$ Translational Medicine Center, The Fourth Affiliated Hospital of China Medical University, Shenyang, Liaoning, China. ${ }^{3}$ Key Laboratory of Molecular Epigenetics, Ministry of Education and Institute of Cytology and Genetics, Northeast Normal University, Changchun, Jilin, China. ${ }^{4}$ Key Laboratory of Polymer Ecomaterials, Changchun Institute of Applied Chemistry Chinese Academy of Sciences, Changchun, Jilin, China.

Received: 1 June 2021 Accepted: 24 August 2021

Published online: 04 September 2021

\section{References}

1. Mertens WC, Bramwell V. Osteosarcoma and other tumors of bone. Curr Opin Oncol. 1994;6:384-90

2. Qu H, Xue Y, Lian W, Wang C, He J, Fu Q, Zhong L, Lin N, Lai L, Ye Z, Wang Q. Melatonin inhibits osteosarcoma stem cells by suppressing SOX9mediated signaling. Life Sci. 2018;207:253-64.

3. Ward E, DeSantis C, Robbins A, Kohler B, Jemal A. Childhood and adolescent cancer statistics, 2014. CA Cancer J Clin. 2014;64:83-103.

4. Mirabello L, Troisi RJ, Savage SA. Osteosarcoma incidence and survival rates from 1973 to 2004: data from the surveillance, epidemiology, and end results program. Cancer. 2009;115:1531-43.

5. Sayles LC, Breese MR, Koehne AL, Leung SG, Lee AG, Liu HY, Spillinger A, Shah AT, Tanasa B, Straessler K, et al. Genome-informed targeted therapy for osteosarcoma. Cancer Discov. 2019;9:46-63.

6. Guerrera MP, Volpe SL, Mao JJ. Therapeutic uses of magnesium. Am Fam Physician. 2009;80:157-62.

7. Wolf Fl, Cittadini A. Magnesium in cell proliferation and differentiation. Front Biosci. 1999:4:D607-17.

8. Al Alawi AM, Majoni SW, Falhammar H. Magnesium and human health: perspectives and research directions. Int J Endocrinol. 2018;2018:9041694

9. Wang W, Yeung KWK. Bone grafts and biomaterials substitutes for bone defect repair: a review. Bioact Mater. 2017;2:224-47.

10. Romani AMP. Beneficial role of $\mathrm{Mg}(2+)$ in prevention and treatment of hypertension. Int J Hypertens. 2018;2018:9013721.

11. Staiger MP, Pietak AM, Huadmai J, Dias G. Magnesium and its alloys as orthopedic biomaterials: a review. Biomaterials. 2006;27:1728-34.

12. Kraus T, Fischerauer SF, Hänzi AC, Uggowitzer PJ, Löffler JF, Weinberg AM. Magnesium alloys for temporary implants in osteosynthesis: in vivo studies of their degradation and interaction with bone. Acta Biomater. 2012;8:1230-8.

13. Li Z, Gu X, Lou S, Zheng Y. The development of binary Mg-Ca alloys for use as biodegradable materials within bone. Biomaterials. 2008;29:1329-44

14. Kamrani S, Fleck C. Biodegradable magnesium alloys as temporary orthopaedic implants: a review. Biometals. 2019;32:185-93.

15. Zhao D, Zhu T, Li J, Cui L, Zhang Z, Zhuang X, Ding J. Poly(lactic-coglycolic acid)-based composite bone-substitute materials. Bioact Mater. 2021;6:346-60.

16. Zhu T, Cui Y, Zhang M, Zhao D, Liu G, Ding J. Engineered three-dimensional scaffolds for enhanced bone regeneration in osteonecrosis. Bioact Mater. 2020:5:584-601.

17. Tian $P, X u D$, Liu X. Mussel-inspired functionalization of PEO/PCL composite coating on a biodegradable AZ31 magnesium alloy. Colloids Surf B Biointerfaces. 2016;141:327-37.

18. Heise S, Virtanen S, Boccaccini AR. Tackling Mg alloy corrosion by natural polymer coatings - a review. J Biomed Mater Res A. 2016;104:2628-41. 
19. Stehle JH, Saade A, Rawashdeh O, Ackermann K, Jilg A, Sebesteny T, Maronde $E$. A survey of molecular details in the human pineal gland in the light of phylogeny, structure, function and chronobiological diseases. J Pineal Res. 2011;51:17-43.

20. Reiter RJ, Tan DX, Fuentes-Broto L. Melatonin: a multitasking molecule. Prog Brain Res. 2010;181:127-51.

21. Zamfir Chiru AA, Popescu CR, Gheorghe DC. Melatonin and cancer. J Med Life. 2014;7:373-4.

22. Fathizadeh $\mathrm{H}$, Mirzaei $\mathrm{H}$, Asemi Z. Melatonin: an anti-tumor agent for osteosarcoma. Cancer Cell Int. 2019;19:319.

23. Maria S, Witt-Enderby PA. Melatonin effects on bone: potential use for the prevention and treatment for osteopenia, osteoporosis, and periodontal disease and for use in bone-grafting procedures. J Pineal Res. 2014;56:115-25.

24. Balci Yuce H, Karatas O, Aydemir Turkal H, Pirim Gorgun E, Ocakli S, Benli I, Cayli S. The effect of melatonin on bone loss, diabetic control, and apoptosis in rats with diabetes with ligature-induced periodontitis. J Periodontol. 2016;87:e35-43.

25. Schaffazick SR, Pohlmann AR, Guterres SS. Nanocapsules, nanoemulsion and nanodispersion containing melatonin: preparation, characterization and stability evaluation. Pharmazie. 2007;62:354-60.

26. Ciani E, Fontaine R, Maugars G, Mizrahi N, Mayer I, Levavi-Sivan B, Weltzien FA. Melatonin receptors in Atlantic salmon stimulate cAMP levels in heterologous cell lines and show season-dependent daily variations in pituitary expression levels. J Pineal Res. 2019;67:e12590.

27. Wen S, Shang Z, Zhu S, Chang C, Niu Y. Androgen receptor enhances entosis, a non-apoptotic cell death, through modulation of Rho/ROCK pathway in prostate cancer cells. Prostate. 2013;73:1306-15.

28. Wang M, Ning X, Chen A, Huang H, Ni C, Zhou C, Yu K, Lan S, Wang Q, Li $S$, et al. Impaired formation of homotypic cell-in-cell structures in human tumor cells lacking alpha-catenin expression. Sci Rep. 2015:5:12223.

29. Overholtzer M, Brugge JS. The cell biology of cell-in-cell structures. Nat Rev Mol Cell Biol. 2008;9:796-809.

30. He MF, Wang S, Wang Y, Wang XN. Modeling cell-in-cell structure into its biological significance. Cell Death Dis. 2013;4:e630.

31. Fais S. Cannibalism: a way to feed on metastatic tumors. Cancer Lett. 2007;258:155-64.

32. Lugini L, Matarrese P, Tinari A, Lozupone F, Federici C, lessi E, Gentile M, Luciani F, Parmiani G, Rivoltini L, et al. Cannibalism of live lymphocytes by human metastatic but not primary melanoma cells. Cancer Res. 2006:66:3629-38.

33. Wang X, Li Y, Li J, Li L, Zhu H, Chen H, Kong R, Wang G, Wang Y, Hu J, Sun B. Cell-in-cell phenomenon and its relationship with tumor microenvironment and tumor progression: a review. Front Cell Dev Biol. 2019;7:311.

34. Fais S, Overholtzer M. Cell-in-cell phenomena in cancer. Nat Rev Cancer. 2018;18:758-66

35. Ma H, Feng C, Chang J, Wu C. 3D-printed bioceramic scaffolds: from bone tissue engineering to tumor therapy. Acta Biomater. 2018;79:37-59.

36. Oksdath Mansilla M, Salazar-Hernandez C, Perrin SL, Scheer KG, Cildir G, Toubia J, Sedivakova K, Tea MN, Lenin S, Ponthier E, et al. 3D-printed microplate inserts for long term high-resolution imaging of live brain organoids. BMC Biomed Eng. 2021;3:6.

37. Zhao W, Zhang W, Ma H, Yang M. NIPA2 regulates osteoblast function by modulating mitophagy in type 2 diabetes osteoporosis. Sci Rep. 2020;10:3078-8

38. Overholtzer M, Mailleux AA, Mouneimne G, Normand G, Schnitt SJ, King RW, Cibas ES, Brugge JS. A nonapoptotic cell death process, entosis, that occurs by cell-in-cell invasion. Cell. 2007;131:966-79.

39. Mizuno N, Abe K, Morishita Y, Yamashita S, Segawa R, Dong J, Moriya T, Hiratsuka M, Hirasawa N. Pentanoic acid induces thymic stromal lymphopoietin production through $\mathrm{G}(\mathrm{q} / 11)$ and Rho-associated protein kinase signaling pathway in keratinocytes. Int Immunopharmacol. 2017;50:216-23.
40. Hobson AD, Judge RA, Aguirre AL, Brown BS, Cui Y, Ding P, Dominguez E, DiGiammarino E, Egan DA, Freiberg GM, et al. Identification of selective dual ROCK1 and ROCK2 inhibitors using structure-based drug design. J Med Chem. 2018;61:11074-100.

41. Chou AJ, Geller DS, Gorlick R. Therapy for osteosarcoma: where do we go from here? Paediatr Drugs. 2008;10:315-27.

42. Siddiqui N, Asawa S, Birru B, Baadhe R, Rao S. PCL-based composite scaffold matrices for tissue engineering applications. Mol Biotechnol. 2018:60:506-32.

43. Zhao S, Xie K, Guo Y, Tan J, Wu J, Yang Y, Fu P, Wang L, Jiang W, Hao Y. Fabrication and biological activity of 3D-printed polycaprolactone/magnesium porous scaffolds for critical size bone defect repair. ACS Biomater Sci Eng. 2020;6:5120-31.

44. Schwarz R, Bruland O, Cassoni A, Schomberg P, Bielack S. The role of radiotherapy in oseosarcoma. Cancer Treat Res. 2009;152:147-64.

45. Errani C, Longhi A, Rossi G, Rimondi E, Biazzo A, Toscano A, Ali N, Ruggieri $P$, Alberghini M, Picci P, et al. Palliative therapy for osteosarcoma. Expert Rev Anticancer Ther. 2011;11:217-27.

46. Gu X, Ding J, Zhang Z, Li Q, Zhuang X, Chen X. Polymeric nanocarriers for drug delivery in osteosarcoma treatment. Curr Pharm Des. 2015;21:5187-97.

47. Chidambaram M, Manavalan R, Kathiresan K. Nanotherapeutics to overcome conventional cancer chemotherapy limitations. J Pharm Pharm Sci. 2011;14:67-77.

48. Altindal DC, Gumusderelioglu M. Melatonin releasing PLGA micro/ nanoparticles and their effect on osteosarcoma cells. J Microencapsul. 2016:33:53-63.

49. Saroia J, Yanen W, Wei Q, Zhang K, Lu T, Zhang B. A review on biocompatibility nature of hydrogels with $3 \mathrm{D}$ printing techniques, tissue engineering application and its future prospective. Bio-Des Manuf. 2018;1:265-79.

50. Liu L, XuY, Reiter RJ. Melatonin inhibits the proliferation of human osteosarcoma cell line MG-63. Bone. 2013;55:432-8.

51. Zhang WL, Meng HZ, Yang RF, Yang MW, Sun GH, Liu JH, Shi PX, Liu F, Yang B. Melatonin suppresses autophagy in type 2 diabetic osteoporosis. Oncotarget. 2016;7:52179-94.

52. Schuster C, Williams LM, Morris A, Morgan PJ, Barrett P. The human MT1 melatonin receptor stimulates CAMP production in the human neuroblastoma cell line SH-SY5Y cells via a calcium-calmodulin signal transduction pathway. J Neuroendocrinol. 2005;17:170-8.

53. Annese V, Bassotti G, Caruso N, De Cosmo S, Gabbrielli A, Modoni S, Frusciante V, Andriulli A. Gastrointestinal motor dysfunction, symptoms, and neuropathy in noninsulin-dependent (type 2) diabetes mellitus. J Clin Gastroenterol. 1999;29:171-7.

54. Wang S, Li L, Zhou Y, He Y, Wei Y, Tao A. Heterotypic cell-in-cell structures in colon cancer can be regulated by IL-6 and lead to tumor immune escape. Exp Cell Res. 2019;382:111447.

55. Liebman E. The function of leucocytes in the growth and regression of the egg of Triturus viridescens. Am J Anat. 2005;77:273-91.

56. Trowell OA. Intracellular lymphocytes in thymus reticular cells and in fibroblasts cultured in vitro. J Physiol. 1949;1 10:5.

57. Ioachim HL. Emperipolesis of lymphoid cells in mixed cultures. Lab Invest. 1965;14:1784-94.

58. Lugini L, Lozupone F, Matarrese P, Funaro C, Luciani F, Malorni W, Rivoltini $L$, Castelli C, Tinari A, Piris A, et al. Potent phagocytic activity discriminates metastatic and primary human malignant melanomas: a key role of ezrin. Lab Invest. 2003;83:1555-67.

\section{Publisher's Note}

Springer Nature remains neutral with regard to jurisdictional claims in published maps and institutional affiliations. 